\title{
Notes on Computational Uncertainties in Probabilistic Risk/Safety Assessment
}

\author{
Antoine Rauzy \\ Department of Mechanical and Production Engineering, Norwegian University of Science and Technology, \\ 7491 Trondheim, Norway; Antoine.Rauzy@ntnu.no; Tel.: +47-918-97-151
}

Received: 5 January 2018; Accepted: 26 February 2018; Published: 4 March 2018

\begin{abstract}
In this article, we study computational uncertainties in probabilistic risk/safety assessment resulting from the computational complexity of calculations of risk indicators. We argue that the risk analyst faces the fundamental epistemic and aleatory uncertainties of risk assessment with a bounded calculation capacity, and that this bounded capacity over-determines both the design of models and the decisions that can be made from models. We sketch a taxonomy of modelling technologies and recall the main computational complexity results. Then, based on a review of state of the art assessment algorithms for fault trees and event trees, we make some methodological proposals aiming at drawing conceptual and practical consequences of bounded calculability.
\end{abstract}

Keywords: probabilistic risk/safety assessment; uncertainties; assessment algorithms; modeling methodologies

\section{Introduction}

A long journey has been made since the WASH 1400 report [1]. Probabilistic risk assessment (PRA) and probabilistic safety assessment (PSA) are nowadays widely accepted and deployed methods to assess risk of industrial systems such as nuclear power plants, offshore platform or aircrafts. Very large models combining fault trees and event trees are routinely used to make decisions about plant design and operations. Powerful tools are available to author and to assess these models.

This does not mean however that the PRA/PSA technology is mature and fully satisfactory. The famous quote by the statistician George Pellam Box "all models are false, some are useful" [2] applies indeed to PRA/PSA models. This statement should be constantly borne in mind when discussing the treatment of uncertainties in these models, which is the topics of the present article. More exactly, the different sources of "falsity" of models should be clearly understood and thoroughly weighted. It is actually questionable to perform long and complex mathematical developments to deal with uncertainties on some particular aspect of the modeling methodology if this aspect is at the end of the day like a drop in the bucket.

In this article, we explain why uncertainties coming from modeling formalisms and assessment algorithms take a very important place in the whole model uncertainty picture. Our experience is that this issue is often underestimated by both scientists and practitioners. This article aims thus at discussing the whys and wherefores of the current situation.

The key point here is that the calculation of probabilistic risk indicators is provably computational hard, namely \#P hard, as demonstrated by Valiant [3] and further completed by Toda [4]. In practice, this means that PRA/PSA models result necessarily of a trade-off between the accuracy of the description of the system under study and the ability to perform calculations on this description. In other words, the risk analyst faces the fundamental epistemic and aleatory uncertainties of risk assessment with a bounded calculation capacity, and this bounded capacity over-determines both the 
design of models and the decisions that can be made from models. With that respect, he or she is like Simon's economical agent who must make decisions with a bounded rationality [5].

The problem at stake can be thus formulated as follows: given my limited modeling and calculation capacities, and given all the uncertainties of the modeling process, where should I concentrate my efforts to ensure a reasonably correct and reasonably robust decision process?

This article is a contribution to answer this vast question. It gives the point of view of a computer scientist. It aims at drawing, from an engineering viewpoint, some consequences of bounded calculability.

The remainder of this article is organized as follows. Section 2 presents a high level view on the PRA/PSA modeling process and tries to locate the different sources of uncertainties. Section 3 establishes a taxonomy of PRA/PSA modeling formalisms and reviews fundamental computational complexity results regarding the calculation of risk indicators for the three categories of models defined by the taxonomy. Section 4 reviews state of the art algorithms for PRA/PSA Boolean model assessment and explains what makes them efficient in practice. Section 5 reports and discusses experimental results on large nuclear PSA models. Finally, Section 6 concludes the article.

\section{The PRA/PSA Modeling Process}

Figure 1 shows an idealized view of the PRA/PSA process. It is worth following it step by step to discuss sources of uncertainties in models.

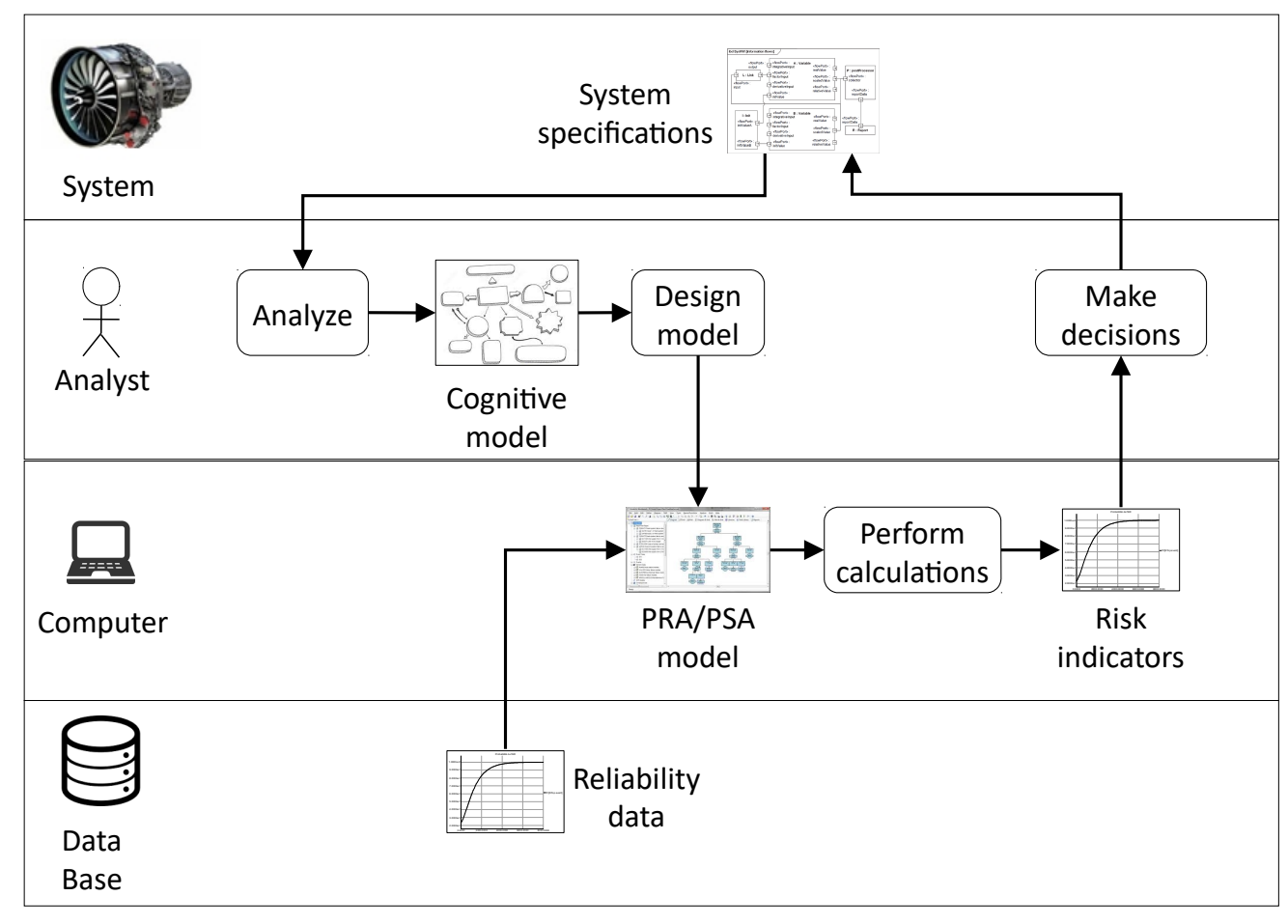

Figure 1. Idealized view of the PRA/PSA process.

The first step of this process consists for the risk analyst in trying to understand how the system works and how it may fail. Functional analysis, as defined in reference textbooks [6,7], is typically part of this step although it does not cover it fully. The risk analyst works usually from system specifications and not from the system itself as the latter (or the configuation under study of the latter) may not exist yet.

One of the fundamental characteristics of risk/ safety assessment is that it is usually not possible to adjust models by means of experiments on the system. Not only the latter may not exist at the moment of the analysis, but also the result of the analysis-roughly speaking the likelihood that something bad happens-is not directly measurable.

The first step is a large source of modeling uncertainties for several reasons including: 
- The physical phenomena at stake may be only partially known and understood.

- The analyst may not master the mathematics (the physics, the chemistry, etc.) of these phenomena.

- System specifications may be incorrect or incomplete.

- The analyst may misunderstand these specifications for they are ambiguous.

- Some initiating events and their consequences within the system may escape the analyst's attention.

- Interrelations between different system components and qualitative predictions of the time behavior in case of the occurrence of initiating events may be mistaken.

These uncertainties are usually called epistemic. This categorization is fine, but one should not forget that risk analyses are performed by individuals with their own knowledge and skills in an industrial process with its own technological and economical constraints. In other words, there may be a significant difference between the body of knowledge that could be relevant for the modeling process and the knowledge the analyst has and is able to use in practice.

The second step of the process consists in designing the actual PRA/PSA model. It reifies (from the Latin: to make thing) the cognitive model into a computerized one. This step takes also reliability data for basic components as input. These reliability data are stored into data bases such as OREDA [8]. The design of the PRA/PSA model is also a large source of uncertainties that must be examined thoroughly.

To design a computerized model, one needs a modeling language, just as to design a computer program one needs a programming language. As of today, most PRA/PSA models are designed using combinatorial modeling formalisms: fault trees, event trees, block diagrams or a combination of those. These formalisms make a strong assumption-the statistical independence of basic eventsand for this reason have strong limitations: impossibility to represent faithfully cold redundancies, time dependencies, resource sharing, reconfigurations, etc. Combinatorial models are thus coarse approximations of behaviors of systems under study. Nevertheless, the use of these formalisms is decided a priori in most of PRA/PSA. Safety standards recommend them. To convince regulation bodies that alternative formalisms could be used is at best a long, a very long process. Consequently, risk analysts tend to reason in terms of combinatorial formalisms, even during the first step of the PRA/PSA process. This is fully understandable, for practical efficiency reasons, but this is also problematic in the sense that this keeps implicit and sometimes even undocumented the knowledge about approximations.

The fault tree/event tree/reliability block diagram methodology requires associating a probability distribution $U_{B E}(t)$ with each basic event $B E$ of the model. Basic events represent failure modes of components of the system. $U_{B E}(t)$ characterizes thus the probability that the component is unavailable at time $t$ in reason of the failure mode described by the basic event $B E$. In industrial practice, most of these probability distributions are either point estimates or parametric distributions-mainly exponential distributions and from time to time Weibull distributions. The parameters of these distributions are obtained from experience feedback on fleets of similar components used in similar conditions. Several important remarks can be made at this point:

- Probability distributions associated with basic events concentrate the aleatory uncertainty about behaviors of systems under study.

- Even if a large experience feedback has been accumulated over the years, the scarcity of reliable data is still an issue. The choice of parametric distributions such as the exponential distribution-which assumes a constant failure rate of the component over its mission time-is often made by default and for the sake of the conveniency rather than supported by strong empirical evidences, see, e.g., the introduction of the already cited OREDA handbook [8] for a discussion.

- Some margins can be taken to deal with the epistemic uncertainty about the aleatory uncertainty by considering probability distributions on parameters of probability distributions associated with basic events. The so-called sensitivity analyses_ implemented in tools such as RiskSpectrum [9] and XFTA [10]—deal with these second order distributions. 
We shall come back to these questions in details in the forthcoming sections.

The third step of the PRA/PSA process consists in calculating risk indicators from the model. Risk indicators include top event probability, importance factors, safety integrity levels and the like (see again reference textbooks [6,7] for a presentation). In most of the commercially available tools, these indicators are calculated from the minimal cutsets. More exactly, approximations of these indicators are calculated from the minimal cutsets. When probabilities of basic events are low and the model is not too large, these approximations are usually very good. When either of these two conditions is missing, results should be handled with care as we shall see in Section 4 .

In any case, calculations of risk indicators are computationally expensive. Moreover, the richer is the modeling formalism, the more expensive is the calculations. This explains why formalisms more expressive than combinatorial formalisms are still seldom used in industrial practice.

In fact, the computational cost of risk indicators has a strong influence back on the whole PRA/PSA process: it determines the choice of modeling formalisms and through this choice the way analysts reason about the system.

The last step of the PRA/PSA process consists in making decisions about the system. These decisions are eventually quite simple: either the risk indicators show that the system is safe and reliable enough to be operated, or some changes have to be made (and the whole PRA/PSA cycle performed again).

In summary, PRA/PSA models have two main roles: first, their design helps risk analysts to review systems, and second, they are means to calculate risk indicators from which decisions can be made. They have several characteristics that make them quite different from models designed in other engineering disciplines:

- $\quad$ They are coarse approximations of the behavior of the system under study.

- It is nearly impossible to adjust them by means of experiments on the real system.

- Their assessment is computationally hard (in a sense we shall make precise in the next section), which over-determines their design and beyond their design, the way analysts reason about the system under study.

Nevertheless, they are the main, if not the only, tool at hand to assess the risk in complex technical systems. In other words, we have to live with epistemic, aleatory and computational uncertainties of risk assessment.

The scientific and technological challenge regarding PRA/PSA is thus to reduce these uncertainties as much as possible, given that modeling and calculation means are necessarily limited. With that respect, a key issue is to ensure that the decision process is reasonably robust, i.e., that small variations in models do not impact these decisions significantly. We shall study how to achieve this objective as efficiently as possible in the forthcoming sections.

\section{The Computational Complexity Barrier}

In this section, we review some important results about the computational complexity of assessment of PRA/PSA models.

\subsection{Taxonomy of Modeling Formalisms}

PRA/PSA models are made of two parts:

- A structural part describes how the system under study may fail under the occurrence of events such as failures, human errors, repairs, reconfigurations, etc.

- A probabilistic part associates probability distributions to the above mentioned events.

The structural part is independent of the probabilistic part.

PRA/PSA modeling formalisms can be divided roughly into three classes according to the expressive power of their structural part: (probabilized) Boolean formulas, (stochastic) finite state automata and (stochastic) process algebras. We shall consider them in turn. 


\subsubsection{Probabilized Boolean Formulas}

Probabilized Boolean formulas include fault trees, event trees, reliability block diagrams (see e.g., [6,7] for reference textbooks) and related formalisms such as Go-Flows [11], Dynamic Flow Graphs [12], multistate systems [13,14], and HiP-HOPS [15].

In these formalisms, the system under study is assumed to consist of a finite number $n$ of components. Each component can be in a finite number of states, usually two (a component is either working or failed). The state the $i$ th component, $1 \leq i \leq n$, is described by means of a variable $v_{i}$ that takes its value into a finite set of constants, like $\{0,1\}$ where 0 stands for working and 1 stands for failed, called the domain of $v_{i}$ and denoted by $\operatorname{dom}\left(v_{i}\right)$. The state of the system is thus described by a vector $\bar{v}=\left\langle v_{1}, \ldots, v_{n}\right\rangle$ of variables that takes its value into the cartesian product $\prod_{i=1}^{n} \operatorname{dom}\left(v_{i}\right)$ of the domains of the variables (which is indeed finite).

The set of states in which the system is failed is described by means of a Boolean formula $f(\bar{v})$ that is interpreted as a subset of $\prod_{i=1}^{n} \operatorname{dom}\left(v_{i}\right)$.

Each variable $v_{i}, 1 \leq i \leq n$, is equipped with a probability distribution, i.e., a function that associates with each value $c \in \operatorname{dom}\left(v_{i}\right)$ and each time $t$ a certain probability $p_{v_{i}=c}(t)$.

It is assumed that components are statistically independent. Therefore, the probability that the system is in state $\bar{s}=\left\langle s_{1}, \ldots, s_{n}\right\rangle$ at time $t$ is simply as follows.

$$
p_{\bar{v}=\bar{s}}(t)=\prod_{i=1}^{n} p_{v_{i}=s_{i}}(t)
$$

From the above definitions, the following equality holds.

$$
p_{f(\bar{s})}(t)=\sum_{\bar{s} \in f(\bar{v})} p_{\bar{v}=\bar{s}}(t)
$$

In theory, $p_{f(\bar{v})}(t)$ is thus easy to assess. In practice, it is impossible to enumerate one by one all of the (failed) states of the system because of the exponential blow-up of their number (more on that point in the next section).

As already pointed out, probabilized Boolean formulas, because they assume components are statistically independent, have strong limitations: impossibility to represent faithfully cold redundancies, time dependencies, repairs, resource sharing, reconfigurations, etc.

\subsubsection{Stochastic Finite State Automata}

Stochastic finite state automata include a large class of modeling formalisms such as Markov chains, (finite) stochastic Petri nets [16], (finite) guarded transition systems [17], dynamic fault trees [18], Boolean driven Markov processes [19], stochastic automata networks [20], stochastic extensions of Harel's StateCharts (see, e.g., [21]) SAML [22], process algebras like PEPA [23] and PEPA-nets [24]... High level modeling languages such as Figaro [25] and AltaRica (in its successive versions: AltaRica LaBRI [26,27], AltaRica Data-Flow [28,29] and AltaRica $3.0[30,31])$ are other and more structured ways to describe finite state automata.

In these formalisms, the state of the system is still described by a vector $\bar{v}=\left\langle v_{1}, \ldots, v_{n}\right\rangle$ of variables that take their values into finite domains $\operatorname{dom}\left(v_{i}\right), 1 \leq i \leq n$. The set of states in which the system is failed is also still described by means of a Boolean formula $f(\bar{v})$ that is interpreted as a subset of $\prod_{i=1}^{n} \operatorname{dom}\left(v_{i}\right)$.

The difference with probabilized Boolean formulas stands in the addition of:

- $\quad$ an initial state $\bar{l}$; and

- a finite set of transitions that describe how the system changes of state under the occurrence of events. 
Transitions are triples $\langle E, g, a\rangle$, denoted $g \stackrel{E}{\rightarrow} a$, where:

- $\quad E$ is the event labeling the transition.

- $\quad g$ is a Boolean formula on the variables of $\bar{v} . g$ is called the guard of the transition.

- $\quad a$ is an instruction that calculates the next values of the variables. $a$ is called the action of the transition.

A transition $g \stackrel{E}{\rightarrow} a$ is fireable in a global state $\bar{s}$ if $g(\bar{s})=$ true. Its firing transforms the state $\bar{s}$ into the state $a(\bar{s})$.

Except for Markov chains, the state space of the system is thus described implicitly: a given state $\bar{t}$ is reachable from the initial state $\bar{l}$ if either $\bar{t}=\bar{l}$, or there is another state $\bar{s}$ and a transition $T: g \stackrel{E}{\rightarrow} a$, such that $\bar{s}$ is reachable from $\bar{l}, T$ is fireable in $\bar{s}$ and $a(\bar{s})=\bar{t}$.

Each event $E$ is equipped with a deterministic or probabilistic delay. The probability to be in the state $\bar{s}$ at time $t$ is thus the sum of the probabilities of all possible sequences of transition firings that lead from state $\bar{l}$ at time 0 to state $\bar{s}$ at time $t$.

Stochastic finite state automata have indeed a much higher expressive power than probabilized Boolean formulas. They make it possible to represent faithfully cold redundancies, time dependencies, repairs, resource sharing, reconfigurations, etc. However, they describe finite state spaces and assume that its architecture does not change throughout its mission.

Note that several above mentioned formalisms entering into the class of stochastic finite state automata make it possible to describe infinite state spaces (e.g., Petri nets). Models are however designed in such way that the state space they describe stays finite.

\subsubsection{Stochastic Process Algebras}

The last class of formalisms, stochastic process algebras, includes formalisms as diverse as (stochastic variants of) colored Petri nets (with an unbound number of colors) [32], process algebras such as Milner's $\pi$-calculus [33], and agent-oriented modeling languages (see, e.g., [34] for an introduction). So-called "Systems of Systems" (see, e.g., [35] for a seminal article) can often be described in this way. Many calculation/simulation models or programming languages have been proposed in the literature that work more or less in this way (Simula has been historically the first one, see, e.g., [36]).

In these formalisms, the state of the system is also described as a vector $\bar{v}$ of variables encoding the individual states of its components and by transitions describing change of states, but:

- Some of the components may be in infinite number of different states (the domains of the corresponding variables are infinite).

- The size of the vector $\bar{v}$ may vary, as new components may be created and some existing components may be destroyed as the result of actions of transitions. The number of transitions may vary as well.

We gave here a presentation of models in terms of automata for the sake of uniformity. It is sometimes easier to see models of this class as descriptions of hierarchical processes running in parallel. Each component of the system is then seen as a process or an agent. During its execution, which may end before the end of the execution of the system as a whole, a process may "fork", i.e., create some sub-processes or clone processes.

Formalisms belonging to this class have the full power of programming languages.

The three classes we mentioned in this section are ordered by increasing computational complexity of assessment algorithms, as we shall see now.

\subsection{Computational Complexity}

Computational complexity theory is a branch of theoretical computer science that aims at classifying problems according to the cost, in terms of computational resources, of solving them. 
We shall recall here only fundamental results related to PRA/PSA. The reader interested in a broader perspective should look at reference textbooks $[37,38]$.

Computational complexity theory considers families of problems stated in mathematical terms. Of course, the cost of solving a problem must be related to the size of this problem. This size of problem can be measured for instance as the number of symbols required to encode this problem. It can be shown that, under reasonable assumptions, this is a suitable measure. The size of an instance $P$ of a problem is denoted $|P|$.

The complexity of a problem is by definition the complexity of the best algorithm to solve that problem. The algorithm should indeed be able to solve any instance of the problem. The complexity of an algorithm is measured in terms of the number of steps this algorithm takes to solve the considered instance of the problem. As this number of steps may vary from one instance to the other, even if we consider instances of the same size, the complexity is characterized by means of a function $f(n)$ such that for any instance of size $n$ of the problem, the number of steps of the algorithm is at most c.f $n$ ), for some predefined constant $c$. It is then said that this algorithm is in $O(f(n))$ (the big-O notation). For instance, sorting the element of a list using the quick-sort algorithm is in $O(n \cdot \log n)$, where $n$ denotes the number of elements of that list.

At this point, three important remarks can be made.

First, one can consider, aside this complexity in terms of the number of steps-called complexity in time- the complexity in terms of number of memory cells required by the algorithm-called complexity in space. Complexity in time provides in general a better understanding of the actual cost of calculations, but we shall see that the complexity in space is usefull as well.

Second, we are speaking here of worst-case complexity. It is also possible to consider average complexity, but results are then much more difficult to establish.

Third, we assumed in the above discussion that the problems at stake are decidable, i.e., that there exist at least one algorithm to solve them. Some important practical problems (for instance the equivalence of two computer programs) are however undecidable, i.e., it can be proven that no general algorithm exists to solve them.

Decidable problems fall in one of the three following categories, with respect to their complexity.

- Provably easy problems, i.e., those for which algorithms with polynomial complexity are known. These problems are said to be P-easy. Some of them are also P-hard, meaning that no algorithm with a lower complexity than polynomial can be designed to solve them.

- Provably hard problems, i.e., those for which it can be proved that any algorithm has at least an exponential complexity. These problems are said to be EXP-hard.

- Problems that are neither provably easy nor provably hard. There is a wide variety of very practical such problems.

The above classification is rather rough as a problem in $O\left(n^{100}\right)$ can hardly be considered as easy in any practical sense. However, very few such problems have been exhibited so far, so the classification is widely accepted.

Until now, we spoke about problems in general. We need now to be more specific and to distinguish decision problems and enumeration problems. A decision problem is a problem with an answer that is either yes or no. An enumeration problem is a problem that consists in counting the number of yes answers of a decision problem or, if a probability structure is defined over the possible answers, in assessing the sum of the probabilities of the yes answers.

Here, another two important remarks can be made.

First, a common point to decision and enumeration problems is that their answer can be encoded in a small space compared to the size of the problem. This is not the case for all of the problems. For instance, the encoding of the set of reachable states of a finite state automaton may be exponentially larger than the encoding of the automaton itself (not to speak about the set of reachable states of a process algebra model that can be infinite while the description of the automaton itself is finite). 
Second, enumeration problems are indeed at least as hard, and in general much harder, than their decision counterpart. If we know how to count the number of solutions to a problem, we know a fortiori if there is a solution to this problem.

\subsection{Complexity of PRA/PSA Assessment}

\subsubsection{The Six Central Problems of PRA/PSA Assessment}

We can now come to the complexity of PRA/PSA assessment. The key risk/safety indicator is indeed the probability that the system is in a failed state at time $t$. The complexity of calculating this probability depends obviously of the class of the model at stake. To characterize this complexity, it is necessary to study also the complexity of the corresponding decision problem. We can thus formulate the following six central problems of PRA/PSA assessment.

SAT: Let $f(\bar{v})$ be a Boolean formula built over a set of variables $\bar{v}$. Is there a valuation $\bar{s}$ of $\bar{v}$ such that $f(\bar{s})=$ true?

RELIABILITY: Let $f(\bar{v})$ be a Boolean formula built over a set of variables $\bar{v}$. Assume moreover that $\bar{v}$ is equipped with a probability structure (as defined above). What is the probability of $f$ (i.e., the sum of probabilities of variable valuations $\bar{s}$ such that $f(\bar{s})=$ true)?

REACHABILITY: Let $M$ be a finite state automaton. Is there a reachable failed state, i.e., is there a sequence of transitions starting from the initial state of $M$ and leading to a failed state?

FSA-RELIABILITY: Let $M$ be a finite state automaton equipped with a probability structure (as defined above). What is the probability to reach a failed state at time $t$ ?

PA-REACHABILITY: Let $M$ be a process algebra model. Is there a reachable failed state, i.e., is there a sequence of transitions starting from the initial state of $M$ and leading to a failed state?

PA-RELIABILITY: Let $M$ be a process algebra model equipped with a probability structure (as defined above). What is the probability to reach a failed state at time $t$ ?

SAT, RELIABILITY and REACHABILITY are "official" names [37,38]. We defined the others for the purpose of the present article.

We shall now review known computational complexity results about the above problems.

\subsubsection{Complexity PRA/PSA Assessment based on Probabilized Boolean Formulas}

SAT plays a central role in computational complexity theory.

A first remark is that it is easy to check whether a candidate variable valuation $\bar{s}$ satisfies $f$ by propagating bottom-up values of variables in the formula. The algorithm to do so is of linear worst case complexity with respect to the size of the formula. The problem is indeed that there are potentially $2^{n}$ valuations to check if $f$ involves $n$ variables (and each variable can take two values).

The class NP is the class of decision problems having the same characteristic as SAT, i.e., such that, given a candidate solution, it is easy to check whether it is actually a solution but there are exponentially many candidate solutions. NP stands for non-deterministic polynomial. Obviously, $P \subseteq N P \subset E X P$.

In 1971, Cook demonstrated the following theorem.

Theorem 1 (Complexity of SAT [39]). SAT is NP-complete, i.e., any problem of the class NP is reducible to SAT, i.e., can be transformed in polynomial time into a SAT instance that has a solution if and only if the problem has one.

The following question is one of the most intriguing of computer science.

$$
\mathrm{P} \stackrel{?}{=} \mathrm{NP}
$$


As of today, it is still open.

Note that MONOTONE-SAT, i.e., the variant of SAT in which the formula $f$ is coherent (monotone), is trivially an easy problem (according to the our classification): it suffices to check whether the valuation that assigns the value true to all variables satisfies $f$ because if $f$ is satisfied by a valuation it must be satisfied by that one as well. We shall give in the next section a formal definition of coherence.

The class \#P (read "sharp P", or "number P") gathers counting and reliability problems associated with NP-hard problem (i.e., problems that are at least as hard as problems in the class NP). For instance, \#SAT is defined as follows.

\#SAT: Let $f$ be a Boolean formula. How many variable valuations satisfy $f$ ?

This class has been introduced by Valiant [3] who showed the following theorem.

Theorem 2 (Complexity of \#SAT [3]). \#SAT is \#P-complete.

The two following additional properties are easy to show (see [38]).

Property 1 (RELIABILITY versus \#SAT). RELIABILITY is at least as hard as \#SAT.

Property 2 (\#MONOTONE-SAT versus \#SAT). \#MONOTONE-SAT, i.e., the problem of counting the number of solutions of a coherent formula, is as hard as \#SAT.

Valiant's theorem has been later completed by Toda.

Theorem 3 (Toda [4]). PP is as Hard as the Polynomial-Time Hierarchy

It would go far beyond the scope of this paper to explain Toda's theorem. Intuitively, it says that if one can count "for free" the number of solutions of a problem, then one is able to solve in polynomial time all of the problems of the polynomial hierarchy, i.e., is very close to be able to solve in polynomial time problems of exponential worst case complexity.

In a word, RELIABILITY is strongly believed to be a hard problem. We shall elaborate further on this topics Section 4 and explain why, despite of these negative results, very large fault trees and related models can be efficiently assessed, thanks to the coherence of models and to suitable approximations.

\subsubsection{Complexity PRA/PSA Assessment based on Stochastic Finite State Automata}

The following theorem establishes the complexity of REACHABILITY.

Theorem 4 (Complexity of REACHABILITY [38]). REACHABILITY is PSPACE-complete.

The above theorem asserts that REACHABILITY can be solved in polynomial space and that any problem in this class can be reduced to REACHABILITY.

The good news is that, despite the fact that there may be a exponential number of reachable states, one can decide in polynomial space whether a failed state is reachable. This result is obtained by accepting to redo some calculations, i.e., pass several times by the same state. The bad news is that the above result is not very useful in practice and that it cannot anyway be applied to the calculation of the probability of being in a failed state at time $t$. The following theorem formalizes this negative result.

Theorem 5 (Complexity of FSA-RELIABILITY). FSA-RELIABILITY is EXP-hard.

The key remark here is that the number of states on sequences leading to failed states may be exponentially large with respect to the size of the problem. FSA-RELIABILITY is thus a hard problem, with all respects. 
As of today, two approaches have been proposed to solve FSA-reliability in practice: the compilation of the model into a Markov chain and stochastic simulation.

A first approach consists thus in compiling, when possible, the model into a Markov chain, and then to apply numerical algorithms to solve Markov chains, see, e.g., [40] for a reference book on these numerical methods and [41] for a study dedicated to reliability models. This approach suffers indeed from the exponential blow-up of the number of states and transitions of the Markov chain. It is however possible to compute approximated Markov chains, with good practical results, see, e.g., [42].

The second approach consists performing Monte-Carlo simulations. Monte-Carlo simulation is the Swiss knife of models engineering in general and reliability engineering in particular, see, e.g., [43] for a recent monograph. It is feasible if the probability to be calculated is not too low (the number of runs required to get reasonably accurate results increases with the inverse of this probability).

In summary, stochastic finite state automata are a reasonable alternative to probabilized Boolean formulas when the system at stake presents characteristics that cannot be faithfully captured by a pure combinatorial model. Assessing stochastic finite state automata is however extremely intensive in terms of calculation resources even if only reasonably good approximations of the values of risk indicators are required. As of today, the use of stochastic finite state automata is thus limited to relatively small models with relatively high values of risk indicators.

\subsubsection{Complexity PRA/PSA Assessment based on Stochastic Process Algebras}

As the reader may expect, the situation gets even worse for process algebra models. Namely, almost any relevant question on these models is undecidable.

Theorem 6 (Complexity of PA-REACHABILITY). PA-REACHABILITY is undecidable.

The above result follows directly from results on severe restrictions of this general problem. For instance, the reachability problem applied to Petri nets with inhibitor arcs is already undecidable [44].

An immediate consequence of the above theorem is that PA-RELIABILITY is also undecidable.

These undecidability results explain probably why process algebras are seldom used for practical reliability studies. The gain in terms of expressive power over stochastic finite state automata is obtained at a too high price.

Note that it is nevertheless still possible to apply to this class of models the approaches developed for stochastic finite state automata, namely the compilation into approximated Markov chains (when possible) and more importantly, stochastic simulation. The author is convinced that this class of models will play an increasingly important role in the future. The key issue however stands in the validation of such models.

\subsection{Wrap-Up}

In this section, we proposed a taxonomy of modeling formalisms that can be used to support PRA/PSA analyses. We review known computational complexity results. They are essentially bad news: assessing risk indicators is an intractable problem, except for the very specific case where the model is coherent fault tree (or an equivalent representation).

This explains why, despite the strong limitations of this class of models, they are almost exclusively used in PRA/PSA practical applications.

We shall study them in further details in the next section.

Before proceeding, we would like to emphasize here that the computational complexity of PRA/PSA assessment is one of the contributors to epistemic uncertainty. It comes in addition to other contributors such as those mentioned in Section 2. The problems raised by computational complexity stand in the impossibility to model the system faithfully because of the complexity of assessments. 


\section{Assessment Algorithms for Probabilized Boolean Formulas}

In this section, we review state of the art assessment algorithms for probabilized Boolean formulas. Understanding how these algorithms work is actually mandatory to handle uncertainties in a proper way. Nevertheless, we shall not enter into technical details, but rather present the principles. In depth presentations can be found in articles by the author $[45,46]$.

\subsection{Taxonomy of Assessment Algorithms}

PRA/PSA models like fault trees, event trees, reliability block diagrams and the like are eventually interpreted as Boolean formulas built over the two constants 0 (false) and 1 (true), a finite set of variables, so-called basic events, and logical connectives "+" (or), "." (and) and "- " (not). Other connectives such as $k$-out-of- $n$ can be easily derived from those.

The calculation of all risk indicators is based on a basic step consisting in calculating the probability of a Boolean formula $f$, given the probabilities of basic events of $f$, which is nothing but the RELIABILITY problem stated in the previous section.

As $f$ may contain repeated events, it is not possible in general to calculate $p(f)$ directly from $f$. $f$ must transformed into an equivalent normalized formula from which the calculation is possible. Two normal forms have been proposed so far: sums-of-minimal-cutsets and binary decision diagrams.

Figure 2 summarizes the calculation flow.

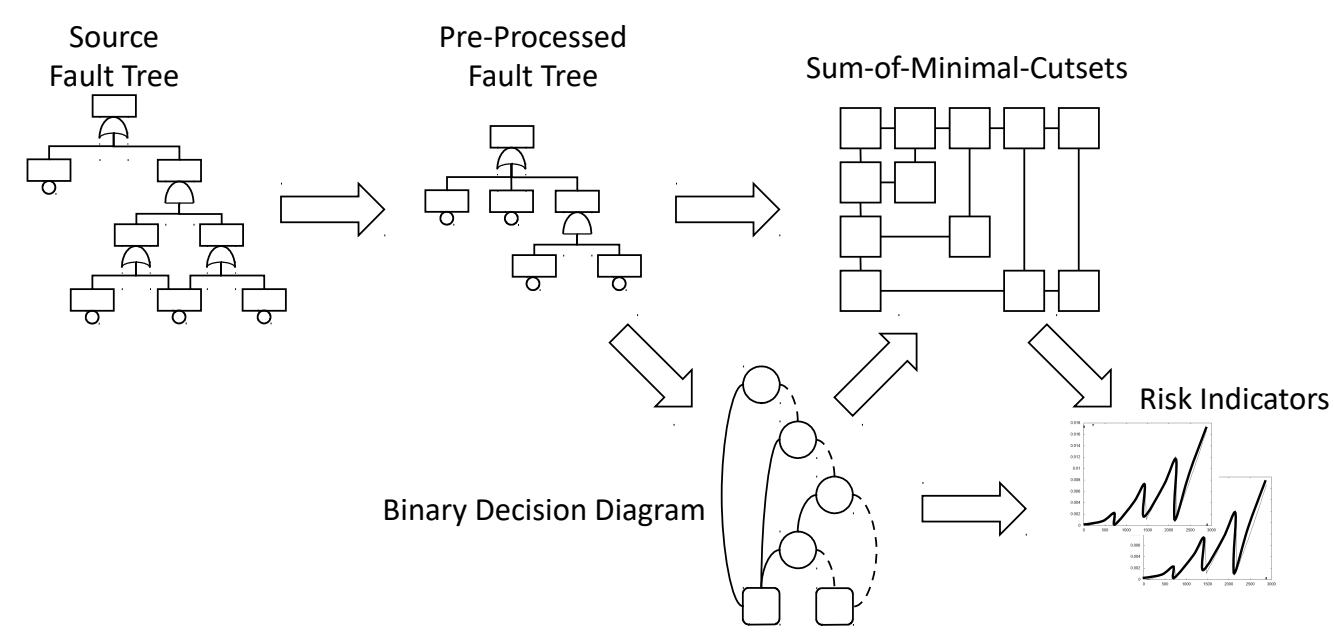

Figure 2. The PRA/PSA calculation flow.

Starting from the initial fault tree (or from the master fault tree generated from a fault tree/event tree model), one pre-processes the model to make it easier to assess. This first step involves notably the detection of modules, i.e., of sub-formulas that are independent from the rest of the model and can thus be assessed separately. The importance of module detection has been pointed out since the very first work on fault tree assessment [47] and is still an essential ingredient of it. Efficient algorithms have been proposed detect modules (e.g., [48]), so the preprocessing phase, although extremely important regarding the overall assessment efficiency, is not itself very resource consuming.

Once the model preprocessed, the hard things start. There is here an alternative with the two above mentioned branches: either a sum-of-minimal-cutsets, or a binary decision diagram is calculated. Minimal cutsets represent failure scenarios. They are of interest on their own, even if no quantification takes place. That is the reason why algorithms have been designed to calculate minimal cutsets from binary decision diagrams [45].

The last step of the assessment consists in calculating risk indicators, either from the sum-of-minimal-cutsets or from the binary decision diagram, depending on which normal form has been chosen. Risk indicators include the top event probability, possibly for different mission times, 
importance factors, safety integrity levels and some others. Efficient algorithms exist to calculate these indicators, see, e.g., Reference [49] for importance factors and Reference [50] for safety integrity levels.

As sum-of-minimal-cutsets and binary decision diagrams play a central role in the whole assessment process, we shall now give more insights about what they are and how they are calculated.

\subsection{Minimal Cutsets}

A literal is either a basic event or its negation. A product is a conjunction of literals that does not contain both a basic event and its negation. A product is positive if it contains no negated basic event. A sum of products is a set of products interpreted as their disjunction. Two products are disjoint if there is at least one basic event occurring positively in one of them and negatively in the other. A sum of disjoint products (SDP) is a sum of products whose products are pair wisely disjoint. A minterm relatively to a set of basic events is a product that contains a literal for each basic event in the set. By construction, two different minterms are disjoint. Minterms one-to-one correspond with truth assignments of basic events (we called them system states in the previous section). For that reason, the following property holds.

Property 3 (Sum-of-Minterms). Any Boolean formula is equivalent to a unique sum of minterms.

Let $f$ and $g$ be two formulas built over the same set of basic events. We denote by Minterms $(f)$ the sum of minterms equivalent to the formula $f$. We say that the minterm $\pi$ satisfies the formula $f$, and denote $\pi \in f$, if $\pi$ belongs to $\operatorname{Minterms}(f)$ and that it falsifies $f$ otherwise. Similarly, we write $f \subseteq g$, if Minterms $(f) \subseteq \operatorname{Minterms}(g)$, i.e., if $f$ entails $g$, and $f \equiv g$ if $\operatorname{Minterms}(f)=\operatorname{Minterms}(g)$, i.e., if $f$ and $g$ are logically equivalent. Note that logical equivalence is the strongest possible equivalence relation over models. Two logically equivalent models are indistinguishable by any correct quantification algorithm.

Let $\pi$ and $\rho$ be two minterms. We say that $\pi$ is smaller than $\rho$, which we denote as $\pi \leq \rho$, if any basic event that occurs positively in $\pi$ occurs positively in $\rho$.

A Boolean formula $f$ is coherent if for any two minterms $\pi$ and $\rho$ such that $\pi \leq \rho, \pi \in f$ implies $\rho \in f$. It is easy to verify that any formula built only over basic events and connectives " + " and "." is coherent.

Let $\pi$ be a positive product. We denote by $\lfloor\pi\rfloor$ the minterm built by completing $\pi$ with the negative literals built over basic events that do not show up in $\pi$. In other words, $\lfloor\pi\rfloor$ is the smallest minterm $\rho$ such that $\rho \in \pi$.

A cutset of a Boolean formula $f$ is defined as a positive product $\pi$, such that $\lfloor\pi\rfloor \in f$. A cutset $\pi$ is minimal if no sub-product of $\pi$ is a cutset of $f$. We shall denote by MCS $(f)$ the set (the sum) of minimal cutsets of the formula $f$. The following property holds [45].

Property 4 (Minimal Cutsets). Let $f$ be a Boolean formula. Then, $f \subseteq M C S(f)$. Moreover:

- $\quad f \equiv M C S(f)$ if and only if $f$ is coherent.

- MCS $(f)$ is the smallest coherent formula containing $f$, i.e., $M C S(f) \subseteq g$ for any coherent formula $g$ such that $f \subseteq g$.

One way of understanding Property 4 is to say that coherent systems are perfectly represented by their minimal cutsets but that for non-coherent systems minimal cutsets are a (sometimes very) conservative approximation of the original model.

Two categories of algorithms have been proposed to calculate minimal cutsets directly from a (pre-processed) fault tree:

- Top-down algorithms, which are derived from MOCUS [51]. Such algorithms are implemented in Risk Spectrum [9] and XFTA [10,52]. 
- Bottom-up algorithms, which use Minato's zero-suppressed binary decision diagrams [53] to encode minimal cutsets. Such an algorithm is implemented in FTREX [54], one of the calculation engines of CAFTA.

In theory, the calculation of the probability of a sum-of-minimal-cutsets can be performed thanks to the Sylvester-Poincaré development.

Property 5 (Sylvester-Poincaré development). Let $f=\sum_{i=1}^{n} \pi_{i}$ be a sum-of-products. Then, the following equality holds.

$$
\begin{aligned}
p(f)= & \sum_{1 \leq i \leq n} p\left(\pi_{i}\right)-\sum_{1 \leq i_{1}<i_{2} \leq n} p\left(\pi_{i_{1}} \cdot \pi_{i_{2}}\right) \\
& +\ldots+-1^{k-1} \sum_{1 \leq i_{1}<\ldots<i_{k} \leq n} p\left(\pi_{i_{1}} \cdot \ldots \cdot \pi_{i_{k}}\right)+\ldots+-1^{n-1} p\left(\pi_{1} \cdot \ldots \cdot \pi_{n}\right)
\end{aligned}
$$

where the probability of a product is the product of the probabilities of its literals.

In practice however, the computational cost of this calculation method is prohibitive as it involves the calculation of $2^{n}$ terms, where $n$ is the number of minimal cutsets. Approximations are thus performed:

- The so-called rare-event approximation that consists in considering only the first term of the development.

$$
p_{R E A}(f) \stackrel{\text { def }}{=} \sum_{\pi \in \operatorname{MCS}(f)} p(\pi)
$$

- The so-called mincut upper bound approximation, which warranties, conversely to the rare-event approximation, to get a result between 0 and 1 .

$$
p_{\text {мсив }}(f) \stackrel{\text { def }}{=} 1-\prod_{\pi \in \operatorname{MCS}(f)}(1-p(\pi))
$$

Both approximations are accurate when the probabilities of basic events are small (say less than $10^{-2}$ ).

\subsection{Binary Decision Diagrams}

Binary decision diagrams are a data structure making it possible to encode in a very compact way the truth table of (many) Boolean functions and to perform operations (conjunction, disjunction, negation, etc.) on these functions. They have been introduced in their modern form by R. Bryant and his colleagues [55,56].

Binary decision diagrams rely on the pivotal or Shannon decomposition.

Property 6 (Pivotal decomposition). Let $f$ be a Boolean formula and $E$ be a basic event (occurring in $f$ ). Then the following equivalence holds.

$$
f \equiv E \cdot f_{E=1}+\bar{E} \cdot f_{E=0}
$$

where $f_{E=v}$ denotes the formula $f$ in which the constant $v$ has been substituted for the basic event $E$.

Technically, binary decision diagrams are directed acyclic graphs with two types of nodes: 
- Leaves $\langle c\rangle$ that are labeled with a Boolean constant $c \in\{0,1\}$. Leaves are interpreted as the constant they are labeled with:

$$
\llbracket\langle c\rangle \rrbracket \stackrel{\text { def }}{=} c
$$

- Internal nodes $\langle E, v, w\rangle$ that are labeled with a basic event $E$ and have two out-edges: a then out-edge pointing to the node $v$, and an else out-edge pointing to the node $w$. Binary decision diagrams are constructed in such a way that the basic event $E$ never shows up in the sub-trees rooted by nodes $v$ and $w$. Internal nodes are interpreted as pivotal decompositions:

$$
\llbracket\langle E, v, w\rangle \rrbracket \stackrel{\text { def }}{=} E \cdot \llbracket v \rrbracket+\bar{E} \cdot \llbracket w \rrbracket
$$

Binary decision diagrams encode thus formulas fully decomposed according to Property 6. They are built bottom-up: the binary decision diagram encoding a formula is obtained by applying Boolean operations on binary decision diagrams encoding its sub-formulas.

Binary decision diagrams have been introduced in the reliability field at the beginning of the nineties [57]. They have proven since then to outperform all other assessment methods-when it is possible to build the binary decision diagram encoding the top event of the fault tree under study. It is not always the case when dealing with large models (with several hundred basic events and more) as the binary decision diagram may be too large to fit into the computer memory (and even on external hard disks).

One of the key features of binary decision diagrams is that they make the calculation of the top event probability both exact (no approximation is required) and of linear complexity, thanks to the following property (that results from Property 6).

Property 7 (Pivotal decomposition applied to probabilities). Let $f$ be a Boolean formula and $E$ be a basic event (occurring in $f$ ). Then the following equivalence holds.

$$
p(f)=p(E) \times p\left(f_{E=1}\right)+(1-p(E)) \times p\left(f_{E=0}\right)
$$

To compute the exact probability of the function represented by means of a binary decision diagram it suffices thus to calculate recursively the probability of each node of the diagram. This principle applies also for the calculation of conditional probabilities and Birnbaum importance factor [49].

\subsection{Consequences of Computational Complexity Results}

Let us summarize the situation by putting together computational complexity results reviewed in the previous section and the algorithms presented above:

1. Fault trees can be assessed in two ways:

- Either by preprocessing the tree, extracting its minimal cutsets and then approximating the top event probability from the minimal cutsets;

- Or by preprocessing the tree, building its binary decision diagram and then calculating the exact top-event probability.

2. RELIABILITY is (strongly believed to be) a hard problem.

3. Preprocessing the tree, approximating the top event probability from the minimal cutsets, and calculating the exact top event probability from the binary decision diagram are easy operations. 
This implies that:

- Either extracting minimal cutsets is a hard problem, or obtaining a good approximation of the top event probability from minimal cutsets is a hard problem, or both.

- Building the binary decision diagram is a hard problem.

These theoretical results are confirmed in practice: the three above operations are actually intractable, at least if we take them in their whole generality.

\subsection{Approximations}

At this point, the reader may think: "All right, this is for the problem in general, but in practice, given the epistemic uncertainties on the system behavior, on its modeling and on reliability data, I'm just fine with reasonable approximations." and she or he is right to think so. The question is: what does mean "reasonable" here?

If no constraint is put on the model, finding accurate approximations seems in fact almost as hard as finding the exact value as demonstrated by several partial results by Ball and Provan, see e.g., [58-60].

However, Boolean PRA/PSA models have two essential characteristics.

First, they are coherent. Even when formulas embeds some negations, these negations are used as a shortcut to represent exclusive configurations and not to reflect a "real" non coherence, see [46] for a discussion. This the reason why they can be assessed by means of minimal cutsets algorithms (which are always coherent). This is not surprising as one can expect that the more components are failed in a mechanical system, the more likely this system is failed. We shall come back on this issue in the next section.

Second, they represent highly reliable systems made of highly reliable components. This translates into the following inequality for most, if not all, of the basic events of the model.

$$
p_{E}(t) \ll p_{\bar{E}}(t)
$$

It follows that large minimal cutsets and minterms with a high number of positive literals have a very low probability and can be safely ignored. In other words, one can focus on failure scenarios involving few faulty components because scenarios involving many faulty components are highly improbable.

These two characteristics are combined into state of the art algorithms to calculate accurate approximations of risk indicators. It works as follows.

First, a probabilistic weight is defined on products as follows. Let $\pi$ be a product.

$$
w(\pi) \stackrel{\text { def }}{=} \prod_{E \in \pi} p(E)
$$

That is, $w(\pi)$ is the product of the probabilities of positive literals of $\pi$.

Now, given a formula $f$ and a probability threshold $\tau$, we can define the following restrictions of $f$ and $\operatorname{MCS}(f)$ with $\tau$ as follows.

$$
\begin{aligned}
& f_{\geq \tau} \stackrel{\text { def }}{=} \sum_{\pi \in \operatorname{Minterms}(f) ; w(\pi) \geq \tau} \pi \\
& \operatorname{MCS}_{\geq \tau}(f) \stackrel{\text { def }}{=}\{\pi \in \operatorname{MCS}(f) ; w(\pi) \geq \tau\}
\end{aligned}
$$

The following property holds. 
Property 8 (Minimal Cutsets of Restrictions [45]). Let $f$ be a Boolean formula and $\tau$ be a probability threshold, then:

$$
\operatorname{MCS}\left(f_{\geq \tau}\right)=M C S_{\geq \tau}(f)
$$

Moreover, under the condition that most of the basic events verify the inequality 3 , the probability of $f$ at time $t$ can be accurately approximated as follows (via the calculation of $\operatorname{MCS}_{\geq \tau}(f)$ ).

$$
\begin{aligned}
& p(f) \approx p_{R E A}\left(f_{\geq \tau}\right) \\
& p(f) \approx p_{M C U B}\left(f_{\geq \tau}\right)
\end{aligned}
$$

Let $p_{l b}$ be the probability of the basic event with lowest probability. Clearly, any product $\pi$ with $k$ positive literals verifies $w(\pi) \geq p_{l b}^{k}$. Therefore, if the cutoff $\tau$ is chosen such that $\tau \leq p_{l b}^{k}$, only minimal cutsets (and minterms) with most $k$ positive literals need to be considered when calculating $p_{R E A}\left(f_{\geq \tau}\right)$ (or $p_{M C U B}\left(f_{\geq \tau}\right)$ ). However, there is only a polynomial number of such products, since there is a polynomial number to select at most $k$ items in a set of $n$ items.

It follows that $p_{R E A}\left(f_{\geq \tau}\right)$ (and $p_{M C U B}\left(f_{\geq \tau}\right)$ ) are polynomial approximations of $p(f)$. They can be calculated via the two alternative algorithmic approaches described above: either by extracting only the minimal cutsets whose probability is higher than $\tau$ or by calculating an approximated binary decision diagram, cutting branches encoding a product $\pi$ such that $w(\pi)<\tau$, see [45] for more details. In both cases, it is possible to track what has been discarded so to get an upper bound of the actual probability.

This very positive result, which makes PRA/PSA of practical interest, should not hide the epistemic problems it raises, due to the following paradox.

Assume we designed a model $M$ at a given level of details. We calculated from $M$ minimal cutsets and relevant risk indicators with a probability threshold $\tau$. As we did our job as correctly as possible, we set up $\tau$ as low as possible for the available calculation power.

Now, assume that for some reason, we decide to refine the model $M$ into a model $M^{\prime}$. $M^{\prime}$ decomposes certain basic events into gates so to analyze with a finer grain the failure modes of some components. A priori, results obtained from $M$ and $M^{\prime}$ should be equivalent. The difference stands in the fact that a minimal cutset of $M$ can be refined into a group of minimal cutsets of $M^{\prime}$.

However, here come two problems. First, as $M^{\prime}$ is larger than $M$ and generates thus possibly many more minimal cutsets than $M$, the probability threshold $\tau$ may be too small for $M^{\prime}$ and the available calculation power. We are thus forced to make the calculations with a coarser probability threshold $\tau^{\prime}$ $\left(\tau^{\prime}>\tau\right)$. Second, a minimal cutset $\pi$ of $M$ whose probability was above to the threshold $\tau$, may be decomposed into several minimal cutsets whose probabilities are below $\tau$ and therefore below $\tau^{\prime}$. It follows that these minimal cutsets will be discarded while assessing $M^{\prime}$.

We are thus in the following paradoxical situation.

Paradox 1 (Model refinement). The more refined is the model, the lower is the risk estimation.

By refining sufficiently the model, we could even make the (evaluated) risk vanish completely.

\subsection{Handling Uncertainties on Reliability Data}

Probability distributions of basic events of PRA/PSA models are known only up to an uncertainty. This problem has many causes, including the scarcity of data, that have been discussed at length in the abundant literature on this topics. We shall not attempt to review these contributions here, as they are not at the core of our subject, but just have a look at how uncertainties are handled in practice when calculating risk indicators.

To simplify the discussion, we shall assume that the mission time of the system is fixed and that probabilities of basic events are calculated at this mission time. Saying that the probability $p(E)$ of 
the basic event $E$ is known only up to an uncertainty, is saying that it belongs to a certain interval $\left[p_{\min }(E), p_{\max }(E)\right]$. The density of probability in this interval has no reason to be uniformly distributed. It can be for instance normally distributed (taking into account truncations due to bounds) arround a certain value.

Assuming given such interval (and density probability within the interval) for each basic event of the model/formula $f$, we can attempt to characterize the uncertainty in the calculation of $p(f)$.

The range of variation of the probability of the formula can be significantly larger than the individual range of variations of the probabilities of the basic events. To understand this phenomena, consider a minimal cutset $\pi=E_{1} \cdot \ldots \cdot E_{k}$. Then, $p_{\min }(\pi)=\prod_{i=1}^{k} p_{\min }\left(E_{i}\right)$ and $p_{\max }(\pi)=\prod_{i=1}^{k} p_{\max }\left(E_{i}\right)$. Consequently, if $p_{\min }\left(E_{i}\right)=\rho_{i} \times p_{\max }\left(E_{i}\right)$ for $i=1, \ldots, k$, then $p_{\min }(\pi)=\prod_{i=1}^{k} \rho_{i} \times p_{\max }(\pi)$. The same reasoning applies to each minimal cutset and therefore for $p_{\min }(f)$ and $p_{\max }(f)$. In other words, individual uncertainties multiply.

For this reason, just performing interval calculation gives in general much too coarse results on industrial size models. Two main alternative methods have been proposed: first, to use extended probability theories, such as the Dempster-Shafer theory [61]; and, second, to perform Monte-Carlo simulations on probabilities of basic events. Both methods have their own advantages and drawbacks.

Extended probability theories make it possible to perform calculations efficiently. However, they do not really solve the above problem. Moreover, determining the degree of belief or plausibility of the failure of a component from field data is a quite difficult task.

With that respect, the Monte-Carlo simulation approach seems more practical. However, it is extremely consuming in terms calculation resources. This is the reason why, simulations are usually performed on the same set of minimal cutsets (or the same binary decision diagram), obtained for a probability threshold $\tau$ and the mean values of basic event probabilities. It would be actually too costly to recompute the minimal cutsets (or the binary decision diagram) for each set of probabilities of basic events.

The next section presents experimental results on industrial use cases that illustrate the different points discussed above.

\section{Experimental Results}

To illustrate the different points discussed in the previous section, we selected three large models out of our benchmarks. These three models comes from the nuclear industry. These models are extracted from PSA studies of an American and two European nuclear power plants (from two different European countries).

The numbers of basic events and gates of these models are as follows.

\begin{tabular}{ccc}
\hline PSA Model & \#Basic Events & \#Gates \\
\hline 1 & 1733 & 1304 \\
2 & 2312 & 5346 \\
3 & 2816 & 5583 \\
\hline
\end{tabular}

Each of these models represents a group of sequences of an event tree model leading to a nuclear accident (e.g., core melt). Models 1 and 2 are non coherent in the sense that they embed negated gates and basic events to represent exclusive or impossible configurations, see, e.g., [46] for a discussion on this issue.

We assessed these models with XFTA [10,52], the fault tree calculation engine the author develops in the framework of the Open-PSA initiative [62,63]. XFTA is a very efficient fault tree calculation engine. It is free of use under unrestrictive conditions.

Experiments reported here have been performed on a PC under Windows 10, with a Intel(R) Core(TM) 64 bits processor cadenced at $2.40 \mathrm{GHz}$ with $8 \mathrm{~GB}$ memory. This PC has been bought at the local supermarket. 


\subsection{Calculation of Minimal Cutsets and the Top-Event Probability}

Tables 1-3 report the results obtained on, respectively, Models 1, 2 and 3. They are organized as follows.

Each row of the table corresponds to a different cutoff value. We took as cutoffs the negative powers of 10, ranging from the first value for which at least one minimal cutset is produced to a value where the top event probability is stabilized.

Note that the critical resource here is the memory rather than the computation time. Thanks to XFTA data structures, it is possible to store about 60 millions minimal cutsets within our computer memory. Beyond, the tool has to be configured specifically, which we did not want to do (we wanted results to be reproducible with the distributed version of XFTA).

The columns of the tables report the following information.

- The first column gives the value of the cutoff.

- The second and third columns give the top event probability computed from the minimal cutsets with respectively the rare event approximation and the mincut upper bound.

- The fourth column gives the number of minimal cutsets.

- The fifth column gives the number of different basic events showing up in the minimal cutsets.

- The sixth column gives, in percentage, the ratio of the value of the rare event approximation obtained for the given threshold and the value of the rare event approximation obtained with the lower cutoff we could calculate with (i.e., the value indicated in the second cell of the last row of the table).

- The seventh column gives, in percentage, the ratio of the number of basic events showing up in the minimal cutsets over the total number of basic events of the model.

- Finally, the eighth column gives the running time in seconds for the whole calculation.

Table 1. Results obtained on Model 1 (1733 basic events, 1304 gates).

\begin{tabular}{cccccccc}
\hline Cutoff & REA & MCUB & \#MCS & \#BE & REA\% & BE\% & Time (s) \\
\hline $1.00 \times 10^{-5}$ & $3.55000 \times 10^{-4}$ & $3.54966 \times 10^{-4}$ & 3 & 4 & $74.50 \%$ & $0.2 \%$ & 0.07 \\
$1.00 \times 10^{-6}$ & $4.03857 \times 10^{-4}$ & $4.03805 \times 10^{-4}$ & 20 & 26 & $84.75 \%$ & $1.5 \%$ & 0.16 \\
$1.00 \times 10^{-7}$ & $4.28640 \times 10^{-4}$ & $4.28578 \times 10^{-4}$ & 122 & 108 & $89.95 \%$ & $6.2 \%$ & 0.42 \\
$1.00 \times 10^{-8}$ & $4.50211 \times 10^{-4}$ & $4.50139 \times 10^{-4}$ & 924 & 237 & $94.48 \%$ & $13.7 \%$ & 1.01 \\
$1.00 \times 10^{-9}$ & $4.65220 \times 10^{-4}$ & $4.65141 \times 10^{-4}$ & 6120 & 429 & $97.63 \%$ & $24.8 \%$ & 2.34 \\
$1.00 \times 10^{-10}$ & $4.71964 \times 10^{-4}$ & $4.71882 \times 10^{-4}$ & 29,098 & 755 & $99.04 \%$ & $43.6 \%$ & 5.39 \\
$1.00 \times 10^{-11}$ & $4.74889 \times 10^{-4}$ & $4.74805 \times 10^{-4}$ & 124,582 & 1055 & $99.66 \%$ & $60.9 \%$ & 12.62 \\
$1.00 \times 10^{-12}$ & $4.75985 \times 10^{-4}$ & $4.75901 \times 10^{-4}$ & 480,930 & 1166 & $99.89 \%$ & $67.3 \%$ & 27.59 \\
$1.00 \times 10^{-13}$ & $4.76365 \times 10^{-4}$ & $4.76281 \times 10^{-4}$ & $1,693,755$ & 1323 & $99.97 \%$ & $76.3 \%$ & 61.00 \\
$1.00 \times 10^{-14}$ & $4.76491 \times 10^{-4}$ & $4.76407 \times 10^{-4}$ & $5,658,636$ & 1464 & $99.99 \%$ & $84.5 \%$ & 137.00 \\
$1.00 \times 10^{-15}$ & $4.76529 \times 10^{-4}$ & $4.76445 \times 10^{-4}$ & $17,579,596$ & 1515 & $100.00 \%$ & $87.4 \%$ & 288.00 \\
\hline
\end{tabular}

Table 2. Results obtained on Model 2 (2312 basic events, 5346 gates).

\begin{tabular}{cccccccc}
\hline Cutoff & REA & MCUB & \#MCS & \#BE & REA\% & BE\% & Time (s) \\
\hline $1.00 \times 10^{-7}$ & $6.48254 \times 10^{-7}$ & $6.48254 \times 10^{-7}$ & 4 & 11 & $11.41 \%$ & $0.5 \%$ & 0.18 \\
$1.00 \times 10^{-8}$ & $2.11285 \times 10^{-6}$ & $2.11284 \times 10^{-6}$ & 57 & 41 & $37.20 \%$ & $1.8 \%$ & 0.39 \\
$1.00 \times 10^{-9}$ & $3.40600 \times 10^{-6}$ & $3.40599 \times 10^{-6}$ & 590 & 149 & $59.96 \%$ & $6.4 \%$ & 0.89 \\
$1.00 \times 10^{-10}$ & $4.40506 \times 10^{-6}$ & $4.40505 \times 10^{-6}$ & 4222 & 348 & $77.55 \%$ & $15.1 \%$ & 2.20 \\
$1.00 \times 10^{-11}$ & $5.07637 \times 10^{-6}$ & $5.07636 \times 10^{-6}$ & 27,543 & 687 & $89.37 \%$ & $29.7 \%$ & 6.03 \\
$1.00 \times 10^{-12}$ & $5.42694 \times 10^{-6}$ & $5.42693 \times 10^{-6}$ & 146,831 & 1095 & $95.54 \%$ & $47.4 \%$ & 15.77 \\
$1.00 \times 10^{-13}$ & $5.58671 \times 10^{-6}$ & $5.58670 \times 10^{-6}$ & 682,050 & 1464 & $98.35 \%$ & $63.3 \%$ & 39.99 \\
$1.00 \times 10^{-14}$ & $5.65404 \times 10^{-6}$ & $5.65403 \times 10^{-6}$ & $2,908,473$ & 1711 & $99.54 \%$ & $74.0 \%$ & 104.00 \\
$1.00 \times 10^{-15}$ & $5.68026 \times 10^{-6}$ & $5.68024 \times 10^{-6}$ & $11,459,524$ & 1919 & $100.00 \%$ & $83.0 \%$ & 280.00 \\
\hline
\end{tabular}


Table 3. Results obtained on Model 3 (2816 basic events, 5583 gates).

\begin{tabular}{cccccccc}
\hline Cutoff & REA & MCUB & \#MCS & \#BE & REA\% & BE\% & Time (s) \\
\hline $1.00 \times 10^{-7}$ & $7.31207 \times 10^{-7}$ & $7.31207 \times 10^{-7}$ & 3 & 6 & $18.51 \%$ & $0.2 \%$ & 0.40 \\
$1.00 \times 10^{-8}$ & $2.00813 \times 10^{-6}$ & $2.00812 \times 10^{-6}$ & 55 & 76 & $50.84 \%$ & $2.7 \%$ & 0.76 \\
$1.00 \times 10^{-9}$ & $3.08379 \times 10^{-6}$ & $3.08379 \times 10^{-6}$ & 457 & 243 & $78.07 \%$ & $8.6 \%$ & 1.14 \\
$1.00 \times 10^{-10}$ & $3.62022 \times 10^{-6}$ & $3.62022 \times 10^{-6}$ & 2421 & 495 & $91.65 \%$ & $17.6 \%$ & 2.60 \\
$1.00 \times 10^{-11}$ & $3.83641 \times 10^{-6}$ & $3.83640 \times 10^{-6}$ & 10,005 & 912 & $97.13 \%$ & $32.4 \%$ & 4.11 \\
$1.00 \times 10^{-12}$ & $3.91496 \times 10^{-6}$ & $3.91495 \times 10^{-6}$ & 36,717 & 1301 & $99.12 \%$ & $46.2 \%$ & 7.56 \\
$1.00 \times 10^{-13}$ & $3.94020 \times 10^{-6}$ & $3.94019 \times 10^{-6}$ & 119,767 & 1577 & $99.75 \%$ & $56.0 \%$ & 14.29 \\
$1.00 \times 10^{-14}$ & $3.94738 \times 10^{-6}$ & $3.94737 \times 10^{-6}$ & 350,488 & 1797 & $99.94 \%$ & $63.8 \%$ & 27.97 \\
$1.00 \times 10^{-15}$ & $3.94930 \times 10^{-6}$ & $3.94929 \times 10^{-6}$ & 958,104 & 1955 & $99.98 \%$ & $69.4 \%$ & 52.00 \\
$1.00 \times 10^{-16}$ & $3.94979 \times 10^{-6}$ & $3.94977 \times 10^{-6}$ & $2,473,798$ & 2084 & $100.00 \%$ & $74.0 \%$ & 98.00 \\
$1.00 \times 10^{-17}$ & $3.94990 \times 10^{-6}$ & $3.94984 \times 10^{-6}$ & $6,074,179$ & 2179 & $100.00 \%$ & $77.4 \%$ & 182.00 \\
\hline
\end{tabular}

We can already draw several important conclusions from this first series of experiments.

First, XFTA is very efficient. It makes it possible to assess very large models, with millions of minimal cutsets, within seconds where other tools take minutes, if not hours, and are not able to compute with cutoffs as low as reported here. At a first glance, this may seem in contradiction with the development we made throughout this article. However, it is not, or not fully. On the one hand, XFTA results of decades of intensive research on algorithm and heuristics. On the other hand, models under study are nearly coherent Boolean formulas for which polynomial time approximations exist, as explained in the previous section. We shall discuss this issue in more details later in the section.

Second, there is not much difference between the results provided by rare event approximation and those obtained with the mincut upper bound. This is due to the fact that minimal cutsets have low probabilities. The benefit of using the latter approximation is thus limited (especially if we balance it with its algorithmic cost).

Third, in the three models, very few minimal cutsets and thus very few basic events, concentrate the most part of the accident probability. Moreover, even when calculating with a very low cutoff value, a significant part of the basic events does not show up in the minimal cutsets. In other words, there is a significant difference between the model as designed and the model as calculated. This calls for the development of tools that would synthesize the calculated model from the designed model and the list of basic events showing up in the minimal cutsets. This means also that the efforts to reduce uncertainties should probably be focused on these few important minimal cutsets and their basic events.

Fourth, the number of minimal cutsets grows steadily as the cutoff decreases. The minimal cutsets with a low probability do not contribute much to the top event probability. However, they have a strong impact on other risk measures like importance measures. Importance measures such as the Birnbaum importance factor, the Risk Achievement Worth and the Risk Reduction Worth, which are extensively used in nuclear PSA studies, discard the probability of the basic event they are measuring, see [49] for a detailed discussion about this topics. Some authors criticized them for this very reason, see, e.g., [64]. However, the key point here is that the ranking of basic events may show a chaotic behavior with respect to the selected cutoff value. This phenomenon has been first pointed out in Reference [65] and confirmed on a larger extent by Duflot et al. [66,67].

\subsection{Testing the Robustness of the Results}

Testing the robustness of the results is indeed of primary importance when assessing the safety of a critical system. This applies especially to the robustness of the assessment of the top event probability, given the existing uncertainties on reliability data, i.e., on probabilities of basic events (or parameters of probability distributions from which these probabilities are obtained). 
As pointed out in the previous section, there are several methods to do so, including interval calculations, interpretation of probabilities into an extended logic (such as the Dempster-Shafer theory), and Monte-Carlo simulation.

As we are seeking here for general results, we shall adopt a slightly different approach. The idea is to study the impact of a variation in the same direction of the probability of all basic events. This method is probably a good way to test the robustness of the results obtained with nominal probabilities of basic events.

A first test consists in making probabilities of basic events vary slightly. Tables 4-6 report results obtained by increasing by $10 \%$ the probabilities of basic events of the three models.

Table 4. Results obtained by increasing by $10 \%$ the probabilities of basic events of Model 1.

\begin{tabular}{cccccccc}
\hline Cutoff & REA & MCUB & \#MCS & \#BE & REA\% & BE\% & Time (s) \\
\hline $1.00 \times 10^{-5}$ & $4.41529 \times 10^{-4}$ & $4.41474 \times 10^{-4}$ & 4 & 6 & $92.66 \%$ & $0.3 \%$ & 0.08 \\
$1.00 \times 10^{-6}$ & $5.00144 \times 10^{-4}$ & $5.00062 \times 10^{-4}$ & 24 & 31 & $104.96 \%$ & $1.8 \%$ & 0.19 \\
$1.00 \times 10^{-7}$ & $5.39665 \times 10^{-4}$ & $5.39563 \times 10^{-4}$ & 170 & 118 & $113.25 \%$ & $6.8 \%$ & 0.48 \\
$1.00 \times 10^{-8}$ & $5.72057 \times 10^{-4}$ & $5.71937 \times 10^{-4}$ & 1339 & 265 & $120.05 \%$ & $15.3 \%$ & 1.15 \\
$1.00 \times 10^{-9}$ & $5.93737 \times 10^{-4}$ & $5.93604 \times 10^{-4}$ & 8579 & 473 & $124.60 \%$ & $27.3 \%$ & 2.73 \\
$1.00 \times 10^{-10}$ & $6.03324 \times 10^{-4}$ & $6.03185 \times 10^{-4}$ & 41,377 & 827 & $126.61 \%$ & $47.7 \%$ & 6.41 \\
$1.00 \times 10^{-11}$ & $6.07380 \times 10^{-4}$ & $6.07239 \times 10^{-4}$ & 173,891 & 1082 & $127.46 \%$ & $62.4 \%$ & 14.95 \\
$1.00 \times 10^{-12}$ & $6.08905 \times 10^{-4}$ & $6.08763 \times 10^{-4}$ & 667,433 & 1190 & $127.78 \%$ & $68.7 \%$ & 33.69 \\
$1.00 \times 10^{-13}$ & $6.09427 \times 10^{-4}$ & $6.09285 \times 10^{-4}$ & $2,345,094$ & 1351 & $127.89 \%$ & $78.0 \%$ & 75.00 \\
$1.00 \times 10^{-14}$ & $6.09599 \times 10^{-4}$ & $6.09456 \times 10^{-4}$ & $7,707,230$ & 1489 & $127.92 \%$ & $85.9 \%$ & 166.00 \\
$1.00 \times 10^{-15}$ & $6.09650 \times 10^{-4}$ & $6.09508 \times 10^{-4}$ & $23,883,995$ & 1523 & $127.94 \%$ & $87.9 \%$ & 352.00 \\
\hline
\end{tabular}

Table 5. Results obtained by increasing by $10 \%$ the probabilities of basic events of Model 2 .

\begin{tabular}{cccccccc}
\hline Cutoff & REA & MCUB & \#MCS & \#BE & REA\% & BE\% & Time (s) \\
\hline $1.00 \times 10^{-7}$ & $1.20217 \times 10^{-6}$ & $1.20217 \times 10^{-6}$ & 6 & 12 & $21.16 \%$ & $0.5 \%$ & 0.20 \\
$1.00 \times 10^{-8}$ & $3.45096 \times 10^{-6}$ & $3.45095 \times 10^{-6}$ & 80 & 48 & $60.75 \%$ & $2.1 \%$ & 0.45 \\
$1.00 \times 10^{-9}$ & $5.73216 \times 10^{-6}$ & $5.73214 \times 10^{-6}$ & 954 & 193 & $100.91 \%$ & $8.3 \%$ & 1.08 \\
$1.00 \times 10^{-10}$ & $7.32196 \times 10^{-6}$ & $7.32193 \times 10^{-6}$ & 7002 & 428 & $128.90 \%$ & $18.5 \%$ & 2.86 \\
$1.00 \times 10^{-11}$ & $8.33469 \times 10^{-6}$ & $8.33465 \times 10^{-6}$ & 42,736 & 766 & $146.73 \%$ & $33.1 \%$ & 7.94 \\
$1.00 \times 10^{-12}$ & $8.86488 \times 10^{-6}$ & $8.86484 \times 10^{-6}$ & 222,655 & 1172 & $156.06 \%$ & $50.7 \%$ & 20.51 \\
$1.00 \times 10^{-13}$ & $9.10702 \times 10^{-6}$ & $9.10699 \times 10^{-6}$ & $1,030,887$ & 1529 & $160.33 \%$ & $66.1 \%$ & 52.81 \\
$1.00 \times 10^{-14}$ & $9.20761 \times 10^{-6}$ & $9.20757 \times 10^{-6}$ & $4,358,927$ & 1775 & $162.10 \%$ & $76.8 \%$ & 140.00 \\
$1.00 \times 10^{-15}$ & $9.24656 \times 10^{-6}$ & $9.24652 \times 10^{-6}$ & $17,060,713$ & 1946 & $162.78 \%$ & $84.2 \%$ & 390.00 \\
\hline
\end{tabular}

Table 6. Results obtained by increasing by $10 \%$ the probabilities of basic events of Model 3 .

\begin{tabular}{cccccccc}
\hline Cutoff & REA & MCUB & \#MCS & \#BE & REA\% & BE\% & Time (s) \\
\hline $1.00 \times 10^{-7}$ & $1.10523 \times 10^{-6}$ & $1.10523 \times 10^{-6}$ & 4 & 11 & $27.98 \%$ & $0.4 \%$ & 0.44 \\
$1.00 \times 10^{-8}$ & $2.87116 \times 10^{-6}$ & $2.87115 \times 10^{-6}$ & 71 & 97 & $72.69 \%$ & $3.4 \%$ & 0.69 \\
$1.00 \times 10^{-9}$ & $4.26522 \times 10^{-6}$ & $4.26521 \times 10^{-6}$ & 560 & 269 & $107.98 \%$ & $9.6 \%$ & 1.28 \\
$1.00 \times 10^{-10}$ & $4.99193 \times 10^{-6}$ & $4.99191 \times 10^{-6}$ & 2982 & 522 & $126.38 \%$ & $18.5 \%$ & 2.39 \\
$1.00 \times 10^{-11}$ & $5.28019 \times 10^{-6}$ & $5.28017 \times 10^{-6}$ & 12,362 & 962 & $133.68 \%$ & $34.2 \%$ & 4.48 \\
$1.00 \times 10^{-12}$ & $5.38284 \times 10^{-6}$ & $5.38283 \times 10^{-6}$ & 45,166 & 1336 & $136.28 \%$ & $47.4 \%$ & 8.38 \\
$1.00 \times 10^{-13}$ & $5.41505 \times 10^{-6}$ & $5.41504 \times 10^{-6}$ & 145,340 & 1612 & $137.09 \%$ & $57.2 \%$ & 16.07 \\
$1.00 \times 10^{-14}$ & $5.42414 \times 10^{-6}$ & $5.42414 \times 10^{-6}$ & 423,962 & 1816 & $137.32 \%$ & $64.5 \%$ & 30.79 \\
$1.00 \times 10^{-15}$ & $5.42654 \times 10^{-6}$ & $5.42653 \times 10^{-6}$ & $1,156,010$ & 1974 & $137.38 \%$ & $70.1 \%$ & 57.47 \\
$1.00 \times 10^{-16}$ & $5.42715 \times 10^{-6}$ & $5.42713 \times 10^{-6}$ & $2,987,579$ & 2098 & $137.40 \%$ & $74.5 \%$ & 110.00 \\
$1.00 \times 10^{-17}$ & $5.42730 \times 10^{-6}$ & $5.42722 \times 10^{-6}$ & $7,320,431$ & 2192 & $137.40 \%$ & $77.8 \%$ & 205.00 \\
\hline
\end{tabular}


These tables are organized as previously. The only difference stands in the sixth column: the reference probability, i.e., the denominator of the ratio, is the one of the previous table so to make clear the difference on the top event probability induced by the slight increase of basic event probabilities.

The probability of the top event is not very impacted by this slight change in basic event probabilities. The increases are respectively of $30 \%, 60 \%$ and $40 \%$.

The numbers of minimal cutsets for each value of the cutoff vary in a similar way. There is an increase, but this increase is not too drastic.

Note that the increase in the top event probability is mostly due to the increase in basic event probabilities and not to the increase in the number of minimal cutsets, at least for the smallest values of the threshold.

The picture changes radically when we consider a more significant change of basic events probabilities. Tables 7-9 report results obtained by multiplying by 2 the probabilities of basic events of the three models. Note that such a variation, although very significant, is not unrealistic given the epistemic uncertainties on these probabilities.

Table 7. Results obtained by multiplying by 2 the probabilities of basic events of Model 1 .

\begin{tabular}{cccccccc}
\hline Cutoff & REA & MCUB & \#MCS & \#BE & REA \% & BE\% & Time (s) \\
\hline $1.00 \times 10^{-5}$ & $2.56231 \times 10^{-3}$ & $2.55952 \times 10^{-3}$ & 49 & 43 & $537.70 \%$ & $2.5 \%$ & 0.18 \\
$1.00 \times 10^{-6}$ & $3.44500 \times 10^{-3}$ & $3.43956 \times 10^{-3}$ & 400 & 145 & $722.94 \%$ & $8.4 \%$ & 0.48 \\
$1.00 \times 10^{-7}$ & $4.20053 \times 10^{-3}$ & $4.19221 \times 10^{-3}$ & 3210 & 301 & $881.48 \%$ & $17.4 \%$ & 1.39 \\
$1.00 \times 10^{-8}$ & $4.66314 \times 10^{-3}$ & $4.65277 \times 10^{-3}$ & 19,527 & 586 & $978.56 \%$ & $33.8 \%$ & 3.41 \\
$1.00 \times 10^{-9}$ & $4.89122 \times 10^{-3}$ & $4.87977 \times 10^{-3}$ & 96,421 & 888 & $1026.43 \%$ & $51.2 \%$ & 8.51 \\
$1.00 \times 10^{-10}$ & $4.98920 \times 10^{-3}$ & $4.97727 \times 10^{-3}$ & 419,437 & 1138 & $1046.99 \%$ & $65.7 \%$ & 22.03 \\
$1.00 \times 10^{-11}$ & $5.02556 \times 10^{-3}$ & $5.01344 \times 10^{-3}$ & $1,603,024$ & 1285 & $1054.62 \%$ & $74.1 \%$ & 52.51 \\
$1.00 \times 10^{-12}$ & $5.03848 \times 10^{-3}$ & $5.02629 \times 10^{-3}$ & $5,706,077$ & 1438 & $1057.33 \%$ & $83.0 \%$ & 128.00 \\
$1.00 \times 10^{-13}$ & $5.04259 \times 10^{-3}$ & $5.03038 \times 10^{-3}$ & $18,723,478$ & 1516 & $1058.19 \%$ & $87.5 \%$ & 291.00 \\
$1.00 \times 10^{-14}$ & $5.04383 \times 10^{-3}$ & $5.03162 \times 10^{-3}$ & $57,063,870$ & 1553 & $1058.45 \%$ & $89.6 \%$ & 677.00 \\
\hline
\end{tabular}

Table 8. Results obtained by multiplying by 2 the probabilities of basic events of Model 2 .

\begin{tabular}{cccccccc}
\hline Cutoff & REA & MCUB & \#MCS & \#BE & REA\% & BE\% & Time (s) \\
\hline $1.00 \times 10^{-7}$ & $4.09621 \times 10^{-5}$ & $4.09613 \times 10^{-5}$ & 113 & 46 & $721.13 \%$ & $2.0 \%$ & 0.47 \\
$1.00 \times 10^{-8}$ & $7.85630 \times 10^{-5}$ & $7.85599 \times 10^{-5}$ & 1529 & 187 & $1383.09 \%$ & $8.1 \%$ & 1.33 \\
$1.00 \times 10^{-9}$ & $1.07583 \times 10^{-4}$ & $1.07577 \times 10^{-4}$ & 12,561 & 485 & $1893.98 \%$ & $21.0 \%$ & 3.79 \\
$1.00 \times 10^{-10}$ & $1.27490 \times 10^{-4}$ & $1.27482 \times 10^{-4}$ & 84,354 & 931 & $2244.44 \%$ & $40.3 \%$ & 11.57 \\
$1.00 \times 10^{-11}$ & $1.38664 \times 10^{-4}$ & $1.38655 \times 10^{-4}$ & 471,371 & 1364 & $2441.16 \%$ & $59.0 \%$ & 33.16 \\
$1.00 \times 10^{-12}$ & $1.44230 \times 10^{-4}$ & $1.44220 \times 10^{-4}$ & $2,357,504$ & 1674 & $2539.14 \%$ & $72.4 \%$ & 97.00 \\
$1.00 \times 10^{-13}$ & $1.46712 \times 10^{-4}$ & $1.46701 \times 10^{-4}$ & $10,620,675$ & 1882 & $2582.84 \%$ & $81.4 \%$ & 296.00 \\
\hline
\end{tabular}

Table 9. Results obtained by multiplying by 2 the probabilities of basic events of Model 3 .

\begin{tabular}{cccccccc}
\hline Cutoff & REA & MCUB & \#MCS & \#BE & REA\% & BE\% & Time (s) \\
\hline $1.00 \times 10^{-7}$ & $2.81395 \times 10^{-5}$ & $2.81391 \times 10^{-5}$ & 84 & 84 & $712.41 \%$ & $3.0 \%$ & 0.59 \\
$1.00 \times 10^{-8}$ & $4.90584 \times 10^{-5}$ & $4.90572 \times 10^{-5}$ & 840 & 232 & $1242.02 \%$ & $8.2 \%$ & 1.06 \\
$1.00 \times 10^{-9}$ & $5.96707 \times 10^{-5}$ & $5.96690 \times 10^{-5}$ & 4355 & 498 & $1510.69 \%$ & $17.7 \%$ & 2.13 \\
$1.00 \times 10^{-10}$ & $6.38861 \times 10^{-5}$ & $6.38841 \times 10^{-5}$ & 18,007 & 869 & $1617.41 \%$ & $30.9 \%$ & 4.18 \\
$1.00 \times 10^{-11}$ & $6.53132 \times 10^{-5}$ & $6.53111 \times 10^{-5}$ & 63,358 & 1295 & $1653.54 \%$ & $46.0 \%$ & 8.30 \\
$1.00 \times 10^{-12}$ & $6.57587 \times 10^{-5}$ & $6.57565 \times 10^{-5}$ & 202,389 & 1608 & $1664.82 \%$ & $57.1 \%$ & 16.96 \\
$1.00 \times 10^{-13}$ & $6.58864 \times 10^{-5}$ & $6.58842 \times 10^{-5}$ & 594,713 & 1818 & $1668.05 \%$ & $64.6 \%$ & 33.93 \\
$1.00 \times 10^{-14}$ & $6.59207 \times 10^{-5}$ & $6.59186 \times 10^{-5}$ & $1,644,065$ & 1977 & $1668.92 \%$ & $70.2 \%$ & 67.00 \\
$1.00 \times 10^{-15}$ & $6.59296 \times 10^{-5}$ & $6.59274 \times 10^{-5}$ & $4,307,856$ & 2108 & $1669.15 \%$ & $74.9 \%$ & 132.00 \\
$1.00 \times 10^{-16}$ & $6.59317 \times 10^{-5}$ & $6.59296 \times 10^{-5}$ & $10,727,093$ & 2207 & $1669.20 \%$ & $78.4 \%$ & 256.00 \\
$1.00 \times 10^{-17}$ & $6.59322 \times 10^{-5}$ & $6.59299 \times 10^{-5}$ & $25,482,478$ & 2271 & $1669.21 \%$ & $80.6 \%$ & 500.00 \\
\hline
\end{tabular}


Now top event probabilities are respectively multiplied by 10, 26 and 17! The number of minimal cutsets is also very significantly bigger for each value of the cutoff. However, as previously, the increase in the top event probability is mostly due to the increase in basic event probabilities and not to the increase in the number of minimal cutsets.

Some calculations that were possible become intractable. In any case, running times are significantly increased.

Note that the same observation applies the other way round as well: if we divide by a factor 2 the probabilities of the basic events, we divide by a factor much greater than 2 the probability of the top event.

Roughly speaking, if we consider, for each basic event $E$ of "mean" probability $p_{E}$ the range $\left[p_{E} / \rho, p_{E} \times \rho\right]$, for a certain factor $\rho \geq 1$, then the top event probability will vary in the interval $\left[p_{\text {top }} / \rho^{k}, p_{\text {top }} \times \rho^{k}\right]$, where $p_{\text {top }}$ is the probability calculated for the mean values of basic event probabilities and $k$ is the "mean" length of minimal cutsets.

This second series of experiments bring good news and bad news. The good news is that is may not be necessary to recompute the minimal cutsets in each run of a Monte-Carlo simulation (on basic event probabilities). Just recomputing the top event probability from the minimal cutsets calculated with the mean values of basic event probabilities is probably sufficient. The bad news is that, if the uncertainties on basic event probabilities are not small, the uncertainty in the top event probability may be so large that this central indicator looses it significance. In this case, other methods (than Monte-Carlo simulation or interval calculation) have to be put in place. A good idea is probably to perform a case study on the probability of the most important basic events. This is fairly possible because, as we have shown, there are not so many such basic events.

\subsection{Discussion}

The results given in this section are puzzling and lead to the following paradox.

Paradox 2 (Feasibility of calculations). Although involving the resolution of theoretically intractable problems, state of the art cutoff based algorithms make it possible to assess very large PRA/PSA models.

We could take this paradox just as another illustration of the famous quote: "In theory there is no difference between theory and practice. In practice there is". However, this is indeed rather unsatisfying, especially because it is easy to exhibit trivial formulas for which the algorithms do not give any good results: For any value of the cutoff $\tau$, consider the disjunction of $n$ similar basic events whose probability $p$ is lower than $\tau$. Clearly, a cutoff based algorithm detects none of the singleton cutsets and therefore estimates the probability of the formula to 0 . However, by letting $n$ growing, we can make the probability of the formula arbitrarily close to 1, i.e., the error of the algorithm as big as we want.

This calls for a characterization of the formulas for which cutoff based algorithms work. This could work as follows.

Let $f$ be a formula built over a set of variables $\mathcal{V}$ and let $\tau$ be a cutoff value. We can split Minterms $(f)$ into two subsets:

- The set Minterms $\geq \tau(f)$ of minterms whose probabilistic weight is greater or equal to $\tau$.

- The set Minterms $<\tau(f)$ of minterms whose probabilistic weight is less than $\tau$.

The absolute error $\sigma_{\tau}(f)$ and the relative error $\rho_{\tau}(f)$ on the estimation of the probability of $f$ made by a cutoff based algorithm for a given value of $\tau$ can be characterized as follows.

$$
\begin{aligned}
& \sigma_{\tau}(f) \stackrel{\text { def }}{=} p\left(\text { Minterms }_{<\tau}(f)\right) \\
& \rho_{\tau}(f) \stackrel{\text { def }}{=} \frac{p\left(\text { Minterms }_{<\tau}(f)\right)}{p(\text { Minterms }(f))}
\end{aligned}
$$


These measures can be used in two ways: for a given value of the cutoff $\tau$, they characterize the relative and absolute errors made by a cutoff based algorithm, and for a given value $\epsilon$ of the relative or absolute error we are ready to accept, they characterize the value of the cutoff to be used.

This characterization of probabilized Boolean formulas is quite different from other complexity measures proposed in the literature. The Shannon's entropy, as introduced by Shannon in [68], can be used to characterize the amount of information in minterms and therefore in formulas. Intuitively, the elements of Minterms ${ }_{\geq \tau}(f)$ tend to have a low Shannon's entropy while those of Minterms $<\tau(f)$ tend to have a high Shannon's entropy. The problem is indeed that the Shannon's entropy of $f$ considers both Minterms $\geq \tau(f)$ and Minterms $<\tau(f)$, i.e., does not make approximations. For the same reason, trying to characterize approximatable formulas by the size of their normal form (which can be seen as a kind of Kolmogorov complexity) or the computational cost of obtaining it (which can be seen as a kind of Benett's logical depth) is not satisfying, see, e.g., [69] for a reference book on these notions. Eventually, the closest notion one can find is probably the probably approximately correct learning (PAC learning) introduced by Valiant in [70] to ground the computational learning theory, see also [71]. Here, the set of hypotheses would be the set of all possible normal forms for formulas whose minterms have probabilistic weight lower than the cutoff $\tau$ and the concept to be learned would be the normal form for $\operatorname{Minterms}_{\geq \tau}(f)$.

\section{Conclusions}

In this article, we studied the uncertainties in probabilistic risk/safety assessment (PRA/PSA) due to the computational complexity of assessment of risk indicators.

First, we proposed a taxonomy of modeling formalisms used in the PRA/PSA context. We reviewed known complexity results for these formalisms and showed that, except for the very particular case where the support model is a nearly coherent probabilized Boolean formula (i.e., can be translated into a nearly coherent fault trees), calculations at stake are intractable. This comes in some sense as an a posterio theoretical justification of a well established practice. We argued that this is also contributing to a large extent to the epistemic uncertainty on systems under study because this latter class of models does not allow to represent faithfully however important features of systems involving dependencies amongst events.

In a second step, we reviewed state of the art assessment algorithms for the assessment of nearly coherent fault trees and related models. We showed that these algorithms actually calculate polynomial approximations of risk indicators, and, provided the probabilities of basic events are low enough, these approximations are accurate. This good news comes however with an epistemic price that we called the model refinement paradox: the more detailed the model, the lower the risk estimation.

Finally, we reported the results of an experimental study on three large PSA models coming from the nuclear industry. This study showed that in these models at least: (i) a few minimal cutsets (and thus basic events) concentrate the probability of the top event; (ii) the number of extracted minimal cutsets grows steadily with the decrease of the cutoff; and (iii) even for low values of the cutoff, a large proportion of basic events do not show up in the extracted minimal cutsets. This has at least two important consequences in terms of epistemic uncertainty: first, there is a real discrepancy between the model as designed and the model as assessed; and, second, risk indicators such as importance measures may show a chaotic behavior with respect to the selected cutoff. We illustrated finally that, although results are quite robust to small variations of basic event probabilities, the uncertainties on the latter's accumulate. Consequently, even not too large uncertainties on basic event probabilities may produce a large uncertainty on risk indicators.

The above theoretical and experimental results should not be taken as a criticism of the probabilistic approach in reliability engineering. Just the contrary: By better understanding its advantages and possible drawbacks, we delimit better its scope and make it a powerful and trustable tool. With that respect, much remains to do in terms of mathematical, algorithmic and experimental developments, to take a better benefit of this approach. 
Assessing the risk in critical systems is and will remain a complex task. The analyst has definitely to face aleatory and epistemic uncertainties, and to face them with a limited computation power. This echoes in the engineering domain Simon's bounded rationality of economic agents. The question at stake is eventually how to be efficient in the modeling process given our bounded computation resources.

Conflicts of Interest: The author declare no conflict of interest.

\section{References}

1. Rasmussen, N.C. Reactor Safety Study. An Assessment of Accident Risks in U.S. Commercial Nuclear Power Plants; Technical Report WASH 1400, NUREG-75/014; U.S. Nuclear Regulatory Commission: Rockville, MD, USA, 1975.

2. Box, G.P. Robustness in the strategy of scientific model building. In Robustness in Statistics; Launer, R.L., Wilkinson, G.N., Eds.; Academic Press: New York, NY, USA, 1979; pp. 201-236.

3. Valiant, L.G. The Complexity of Enumeration and Reliability Problems. SIAM J. Comput. 1979, 8, 410-421.

4. Toda, S. PP is as Hard as the Polynomial-Time Hierarchy. SIAM J. Comput. 1991, 20, 865-877.

5. Simon, H. Models of Man: Social and Rational. Mathematical Essays on Rational Behavior in a Social Setting; Wiley: New York, NY, USA, 1957.

6. Kumamoto, H.; Henley, E.J. Probabilistic Risk Assessment and Management for Engineers and Scientists; IEEE Press: Piscataway, NJ, USA, 1996.

7. Rausand, M.; Høyland, A. System Reliability Theory: Models, Statistical Methods, and Applications, 2nd ed.; Wiley-Blackwell: Hoboken, NJ, USA, 2004.

8. OREDA Handbook-Offshore Reliability Data, 6th ed.; SINTEF: Trondheim, Norway, 2015; Volumes 1 and 2.

9. Berg, U.; Sardh, H. Risk Spectrum, Theory Manual; RELCON Teknik AB: Sundbyberg, Sweden, 1994.

10. Rauzy, A. XFTA: An Open-PSA Fault-Tree Engine; AltaRica Association: Paris, France, 2014.

11. Matsuoka, T.; Kobayashi, M. GO-FLOW: A new reliability analysis method. Nucl. Sci. Eng. 1988, 98, 64-78.

12. Yau, M.; Apostolakis, G.E.; Guarro, S. The use of prime implicants in dependability analysis of software controlled systems. Reliab. Eng. Sys. Saf. 1998, 62, 23-32.

13. Lisnianski, A.; Levitin, G. Multi-State System Reliability; Quality, Reliability and Engineering Statistics; World Scientific: London, UK, 2003.

14. Natvig, B. Multistate Systems Reliability Theory with Applications; Wiley: Hoboken, NJ, USA, 2010.

15. Adachi, M.; Papadopoulos, Y.; Sharvia, S.; Parker, D.; Tohdo, T. An approach to optimization of fault tolerant architectures using HiP-HOPS. Softw. Pract. Exp. 2011, 41, 1303-1327.

16. Ajmone-Marsan, M.; Balbo, G.; Conte, G.; Donatelli, S.; Franceschinis, G. Modelling with Generalized Stochastic Petri Nets; John Wiley \& Sons, Inc.: New York, NY, USA, 1994.

17. Rauzy, A. Guarded Transition Systems: A new States/Events Formalism for Reliability Studies. J. Risk Reliab. 2008, 222, 495-505.

18. Dugan, J.B.; Bavuso, S.J.; Boyd, M.A. Dynamic fault-tree models for fault-tolerant computer systems. IEEE Trans. Reliab. 1992, 41, 363-377.

19. Bouissou, M.; Bon, J.L. A new formalism that combines advantages of Fault-Trees and Markov models: Boolean logic-Driven Markov Processes. Reliab. Eng. Syst. Saf. 2003, 82, 149-163.

20. Plateau, B.; Stewart, W.J. Stochastic Automata Networks. In Computational Probability; Kluwer Academic Press: Dordrecht, The Netherlands, 1997; pp. 113-152.

21. Jansen, D.N. Extensions of Statecharts: With Probability, Time, and Stochastic Timing. Ph.D. Thesis, University of Twente, Enschede, The Nertheland, October 2003.

22. Güdemann, M.; Ortmeier, F. A Framework for Qualitative and Quantitative Model-Based Safety Analysis. In Proceedings of the IEEE 12th High Assurance System Engineering Symposium (HASE 2010), San Jose, CA, USA, 1-4 November 2010; pp. 132-141.

23. Hillston, J. A Compositional Approach to Performance Modelling; Cambridge University Press: New York, NY, USA, 1996.

24. Gilmore, S.; Hillston, J.; Kloul, L.; Ribaudo, M. PEPA nets: A structured performance modelling formalism. Perform. Eval. 2003, 54, 79-104. 
25. Bouissou, M.; Bouhadana, H.; Bannelier, M.; Villatte, N. Knowledge modelling and reliability processing: Presentation of the FIGARO language and of associated tools. In Proceedings of SAFECOMP'91 - IFAC International Conference on Safety of Computer Control Systems, Trondheim, Norway, 30 October-1 November 1991.

26. Point, G.; Rauzy, A. AltaRica: Constraint automata as a description language. J. Eur. Sys. Autom. 1999, 33, 1033-1052.

27. Arnold, A.; Griffault, A.; Point, G.; Rauzy, A. The AltaRica Formalism for Describing Concurrent Systems. Fundam. Inf. 2000, 34, 109-124.

28. Rauzy, A. Modes Automata and their Compilation into Fault Trees. Reliab. Eng. Syst. Saf. 2002, 78, 1-12.

29. Boiteau, M.; Dutuit, Y.; Rauzy, A.; Signoret, J.P. The AltaRica Data-Flow Language in Use: Assessment of Production Availability of a MultiStates System. Reliab. Eng. Syst. Saf. 2006, 91, 747-755.

30. Prosvirnova, T.; Batteux, M.; Brameret, P.A.; Cherfi, A.; Friedlhuber, T.; Roussel, J.M.; Rauzy, A. The AltaRica 3.0 project for Model-Based Safety Assessment. IFAC Proc. Vol. 2013, 46, 127-132.

31. Prosvirnova, T. AltaRica 3.0: A Model-Based Approach for Safety Analyses. Ph.D. Thesis, Ecole Polytechnique, Paris, France, November 2014.

32. Jensen, K. Coloured Petri Nets; Springer: Berlin/Heidelberg, Germany, 2014.

33. Milner, R. Communicating and Mobile Systems: The Pi-Calculus; Cambridge University Press: Cambridge, UK, 1999.

34. Railsback, S.; Grimm, V. Agent-Based and Individual-Based Modeling-A Practical Introduction; Princeton University Press: Princeton, NJ, USA, 2011.

35. Maier, M.W. Architecting principles for systems-of-systems. Syst. Eng. 1998, 1, 267-284.

36. Kirkerud, B. Object-Oriented Programming With Simula; Addison Wesley: Boston, MA, USA, 1989.

37. Garey, M.R.; Johnson, D.S. Computers and Intractability: A Guide to the Theory of NP-Completeness; Freeman: San Fransisco, CA, USA, 1979.

38. Papadimitriou, C.H. Computational Complexity; Addison Wesley: Boston, MA, USA, 1994.

39. Cook, S. The complexity of theorem proving procedures. In Proceedings of the Third Annual ACM Symposium on Theory of Computing, Shaker Heights, OH, USA, 3-5 May 1971; pp. 151-158.

40. Stewart, W.J. Introduction to the Numerical Solution of Markov Chains; Princeton University Press: Princeton, NJ, USA, 1994.

41. Rauzy, A. An Experimental Study on Six Algorithms to Compute Transient Solutions of Large Markov Systems. Reliab. Eng. Syst. Saf. 2004, 86, 105-115.

42. Brameret, P.A.; Rauzy, A.; Roussel, J.M. Automated generation of partial Markov chain from high level descriptions. Reliab. Eng. Syst. Saf. 2015, 139, 179-187.

43. Zio, E. The Monte Carlo Simulation Method for System Reliability and Risk Analysis; Springer: London, UK, 2013.

44. Esperza, J. Decidability and Complexity of Petri Nets Problems-An introduction. In Lectures on Petri Nets I: Basic Models; Reisig, W., Rozenberg, G., Eds.; Springer: New York, NY, USA, 1998; pp. 374-428.

45. Rauzy, A. Mathematical Foundation of Minimal Cutsets. IEEE Trans. Reliab. 2001, 50, 389-396.

46. Nusbaumer, O.; Rauzy, A. Fault Tree Linking versus Event Tree Linking Approaches: A Reasoned Comparison. J. Risk Reliab. 2013, 227, 315-326.

47. Birnbaum, Z.W.; Esary, J.P. Modules of coherent binary systems. SIAM J. Appl. Math. 1965, 13, $442-462$.

48. Dutuit, Y.; Rauzy, A. A Linear Time Algorithm to Find Modules of Fault Trees. IEEE Trans. Reliab. 1996, 45, 422-425.

49. Dutuit, Y.; Rauzy, A. Importance Factors of Coherent Systems: A Review. J. Risk Reliab. 2013, 228, $313-323$.

50. Innal, F.; Dutuit, Y.; Rauzy, A.; Signoret, J.P. New insight into the average probability of failure on demand and the probability of dangerous failure per hour of safety instrumented systems. Jo. Risk Reliab. 2010, $224,75-86$.

51. Fussel, J.B.; Vesely, W.E. A New Methodology for Obtaining Cut Sets for Fault Trees. Trans. Am. Nucl. Soc. $1972,15,262-263$.

52. Rauzy, A. Anatomy of an Efficient Fault Tree Assessment Engine. In Proceedings of the International Joint Conference PSAM'11/ESREL'12, Helsinki, Finland, 25-29 June 2012. 
53. Minato, S.I. Zero-Suppressed BDDs for Set Manipulation in Combinatorial Problems. In Proceedings of the 30th ACM/IEEE Design Automation Conference, DAC'93, Dallas, TX, USA, 14-18 June 1993; pp. $272-277$.

54. Jung, W.S.; Han, S.H.; Ha, J. A fast BDD algorithm for large coherent fault trees analysis. Reliab. Eng. Syst. Saf. 2004, 83, 369-374.

55. Bryant, R.S. Graph Based Algorithms for Boolean Fonction Manipulation. IEEE Trans. Comput. 1986, 35, 677-691.

56. Brace, K.S.; Rudell, R.L.; Bryant, R.S. Efficient Implementation of a BDD Package. In Proceedings of the 27th ACM/IEEE Design Automation Conference, Orlando, FL, USA, 24-28 June 1990; pp. 40-45.

57. Rauzy, A. New Algorithms for Fault Trees Analysis. Reliab. Eng. Syst. Saf. 1993, 05, 203-211.

58. Ball, M.O. Computational complexity of network reliability analysis: An overview. IEEE Trans. Reliab. 1986, 35, 230-239.

59. Provan, J.S. Bounds on the reliability of networks. IEEE Trans. Reliab. 1986, 35, 260-268.

60. Provan, G.M. The computational complexity of multiple-context truth maintenance system. In Proceedings of the 9th European Conference on Artificial Intelligence, ECAI'90, Stockholm, Sweden, August 1990; Aiello, L.C., Ed.; Pitman Publishing: London, UK; pp. 523-527.

61. Shafer, G. A Mathematical Theory of Evidence; Princeton University Press: Princeton, NJ, USA, 1976.

62. Epstein, S.; Nusbaumer, O.; Rauzy, A.; Wakefield, D. A Modest Proposal: A Standard PSA Model Representation Format. In Proceedings of the International Conference Nuclear Energy for New Europe 2007, Portoroz, Slovenia, 10-13 September 2007.

63. Epstein, S.; Reinhart, M.; Rauzy, A. The open PSA initiative for next generation probabilistic safety assessment. In Proceeding of the 9th International Conference on Probabilistic Safety Assessment and Management 2008, PSAM 2008, Hong Kong, China, 18-23 May 2008; pp. 542-550.

64. Cheok, M.C.; Parry, G.W.; Sherry, R.R. Use of importance measures in risk informed regulatory applications. Reliab. Eng. Syst. Saf. 1998, 60, 213-226.

65. Epstein, S.; Rauzy, A. Can We Trust PRA? Reliab. Eng. Syst. Saf. 2005, 88, 195-205.

66. Duflot, N.; Bérenguer, C.; Dieulle, L.; Vasseur, D. How to build an adequate set of minimal cutsets for PSA importance measure calculation (PSAM-0071). In Proceedings of the Eighth International Conference on Probabilistic Safety Assessment \& Management (PSAM), New Orleans, Louisiana, 14-18 May 2006.

67. Duflot, N.; Bérenguer, C.; Dieulle, L.; Vasseur, D. Calculating importance measures in PSA at different levels. In Safety and Reliability for Managing Risk, Proceedings of the 15th European Safety and Reliability Conference (ESREL 2006), Estoril, Portugal, 18-22 September 2006; Soares, C.G., Zio, E., Eds.; CRC Press: London, UK, 2006; pp. 2405-2412.

68. Shannon, C.E. A Mathematical Theory of Communication. Bell Syst. Tech. J. 1948, 27, 379-423.

69. Li, M.; Vitanyi, P. An Introduction to Kolmogorov Complexity and Its Applications, 3rd ed.; Springer: New York, NY, USA, 2014.

70. Valiant, L.G. A theory of the learnable. Commun. ACM 1984, 27, 1134-1142.

71. Valiant, L.G. Probably Approximately Correct: Nature's Algorithms for Learning and Prospering in a Complex World; Basic Books: New York, NY, USA, 2013.

(C) 2018 by the author. Licensee MDPI, Basel, Switzerland. This article is an open access article distributed under the terms and conditions of the Creative Commons Attribution (CC BY) license (http://creativecommons.org/licenses/by/4.0/). 